ARTICLE OPEN

\title{
A Rift Valley fever virus Gn ectodomain-based DNA vaccine induces a partial protection not improved by APC targeting
}

Tiphany Chrun ${ }^{1,2}$, Sandra Lacôte ${ }^{2}$, Céline Urien ${ }^{1}$, Luc Jouneau ${ }^{1}$, Céline Barc ${ }^{3}$, Edwige Bouguyon ${ }^{1}$, Vanessa Contreras ${ }^{4}$, Audrey Ferrier-Rembert ${ }^{5}$, Christophe N. Peyrefitte ${ }^{5}$, Nuria Busquets ${ }^{6}$, Enric Vidal ${ }^{6}$, Joan Pujols ${ }^{6}$, Philippe Marianneau ${ }^{2}$ and Isabelle Schwartz-Cornil ${ }^{1}$

Rift Valley fever virus, a phlebovirus endemic in Africa, causes serious diseases in ruminants and humans. Due to the high probability of new outbreaks and spread to other continents where competent vectors are present, vaccine development is an urgent priority as no licensed vaccines are available outside areas of endemicity. In this study, we evaluated in sheep the protective immunity induced by DNA vaccines encoding the extracellular portion of the Gn antigen which was either or not targeted to antigen-presenting cells. The DNA encoding untargeted antigen was the most potent at inducing lgG responses, although not neutralizing, and conferred a significant clinical and virological protection upon infectious challenge, superior to DNA vaccines encoding the targeted antigen. A statistical analysis of the challenge parameters supported that the anti-eGn lgG, rather than the Tcell response, was instrumental in protection. Altogether, this work shows that a DNA vaccine encoding the extracellular portion of the $\mathrm{Gn}$ antigen confers substantial-although incomplete-protective immunity in sheep, a natural host with high preclinical relevance, and provides some insights into key immune correlates useful for further vaccine improvements against the Rift Valley fever virus.

npj Vaccines (2018)3:14; doi:10.1038/s41541-018-0052-x

\section{INTRODUCTION}

The Rift Valley Fever Virus (RVFV) is a zoonotic phlebovirus (Phenuiviridae) transmitted by mosquitoes, causing serious diseases in humans and ruminants. In humans, RVFV usually induces a febrile illness, and sometimes hepatitis, hemorrhagic fever, retinitis or encephalitis, leading to death. In ruminants, RVFV is responsible for abortions in pregnant ewes and a high die-off rate in young sheep (see ref. ${ }^{1}$ for review). RVFV has caused recurrent outbreaks in Sub-Saharan Africa since 1931 and later spread to the Arabian Peninsula, raising awareness of the potentiality for introduction to other continents where a large array of competent insects would embed the virus in the ecosystem with devastating health, social and economic consequences. Attenuated and inactivated RVFV vaccines are commercialized for ruminants in enzootic countries. However, the risk of reversion of attenuated vaccines renders their registration unlikely in Europe and the United States and the need for repeated injections of inactivated vaccines is not compatible with herd management in most countries. The 2017 WHO Blueprint list cites RVFV as one of the 10 priority pathogens for which a low-cost, safe, stable and highly effective vaccine is urgently needed (http://www.who.int/ blueprint/priority-diseases/en/).

The $M$ segment of the tripartite negative stranded RVFV RNA genome encodes for a polypeptide generating the $\mathrm{Gn}$ and $\mathrm{Gc}$ glycoproteins which are involved in viral attachment and fusion, respectively. Neutralizing antibodies (NAbs) are directed to both $\mathrm{Gn}$ and $\mathrm{Gc}^{2} \mathrm{Gc}$ is located within the glycoprotein shell whereas
Gn is exposed on the outside, indicating that $\mathrm{Gn}$ is a major target of NAbs. ${ }^{3}$ Importantly, anti-Gn and Gc NAbs can confer protection by passive transfer. ${ }^{4}$ Expression of the $\mathrm{Gn}$ ectodomain allowed production of a secreted form (eGn) that, after injection as a protein vaccine in mice, conferred full protection against RVFV, indicating that $e G n$ is a promising antigen candidate for a subunit vaccine. Furthermore a DNA vaccine encoding $e G n$ was found to be partially protective in mice, despite low levels of NAbs. ${ }^{6}$ Finally, $\mathrm{CD}^{+}{ }^{+} \mathrm{T}$-cell responses against the $\mathrm{Gn} / \mathrm{Gc}$ proteins were identified in the context of protective immune responses, ${ }^{7}$ but their role in protection is unclear.

DNA vaccines present many of the qualities desired for a new RVFV vaccine: they show good stability outside cold chain, they can be prepared for emergency vaccine banks, they are noninfectious and safe, they allow differentiation of infected from vaccinated animals (DIVA), they are easy to construct, their manufacture is feasible at large scale and they have the potential to be cost effective to produce, especially when compared to recombinant proteins. ${ }^{8,9}$ However, whereas DNA vaccines induce high $\mathrm{T}$-cell and $\mathrm{Ab}$ responses in rodent models, they usually display low immunogenicity properties in other mammals including ruminants and humans. ${ }^{10}$ Improvement of DNA vaccine immunogenicity has been achieved by enhancing the in vivo delivery with electroporation in skin and muscle, ${ }^{11}$ by adding genetically encoded adjuvants such as granulocyte-macrophage colony-stimulating factor (GM-CSF), ${ }^{8}$ as well as by fusing the encoded vaccine antigen to a single chain fragment variable

\footnotetext{
${ }^{1}$ VIM-INRA-Université Paris-Saclay, Domaine de Vilvert, 78350 Jouy-en-Josas, France; ${ }^{2}$ ANSES-Laboratoire de Lyon, Unité Virologie, Lyon, France; ${ }^{3}$ UE1277-INRA, Plate-Forme

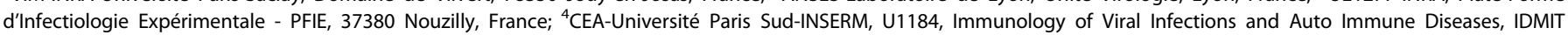

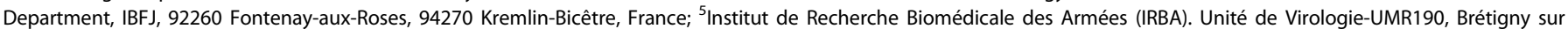
Orge, France and ${ }^{6}$ IRTA, Centre de Recerca en Sanitat Animal (CReSA, IRTA-UAB), Campus de la Universitat Autònoma de Barcelona, 08193 Bellaterra, Spain Correspondence: Isabelle Schwartz-Cornil (isabelle.schwartz@inra.fr)
}

Received: 4 October 2017 Revised: 26 February 2018 Accepted: 28 March 2018 Published online: 20 April 2018 
(scFv), or a chemokine, for targeting a receptor expressed on antigen-presenting cells (APCs). ${ }^{12-16}$ Among APCs, conventional dendritic cells (CDCs) appear as the key cell type involved in APCtargeting efficacy. ${ }^{17}$ In mice cDCs have been classified in two major subsets, the $\mathrm{CDC1}$ and $\mathrm{CDC} 2$, with the $\mathrm{CDC1}$ subset excelling in $\mathrm{CD}^{+} \mathrm{T}$ and $\mathrm{T}$ helper cell type 1 (Th1) $\mathrm{CD} 4^{+} \mathrm{T}$-cell activation and the CDC2 subset promoting the T helper cell type 2 and 17 (Th2 and Th17) $\mathrm{CD}^{+}{ }^{+}$T-cell differentiation. ${ }^{18}$ Homologous $\mathrm{CDC1}$ and CDC2 subsets have been identified in other species, i.e., in humans, monkey, sheep and pig, and they can be distinguished based on a conserved trans-species phenotype with $\mathrm{CDC} 1$ being $C A D M 11^{\text {hi- }}$ $\mathrm{SIRP}^{\mathrm{lo}}$ and $\mathrm{CDC} 2$ being CADM1 ${ }^{\mathrm{lo}} \mathrm{SIRP}^{\text {hi.19,20 }}$ A large number of studies, primarily conducted in mice, revealed that targeting antigens to CDCs using protein or DNA vaccines results in enhancing and even biasing the type of immune responses, depending on both the targeted CDC subset and the nature of the targeted receptor (see refs. ${ }^{21,22}$ for reviews). The most comprehensively studied molecule for APC targeting is DEC $205^{22}$ which is expressed on CDCs in many species including ruminants ${ }^{23}$ and is overexpressed on cDC1s at least in mice. The targeting of different antigens to DEC205 in DNA vaccination of mice ${ }^{12,1324,25}$ resulted in enhanced $\mathrm{Ab}, \mathrm{CD}^{+}$and $\mathrm{CD} 8^{+} \mathrm{T}$-cell responses. In addition in a study comparing different targeted receptors using chimeric protein vaccines, $C D 11 c$, the general marker of $D C$, revealed to be a particularly suitable receptor for inducing high humoral responses ${ }^{26}$ and was also efficient at enhancing both $\mathrm{Ab}$ and $\mathrm{T}$ cell responses with DNA vaccines in mice. ${ }^{14}$ Despite the spectacular promises from mouse models, the improvement of protective immunity by APC-targeting remains to be documented in large mammals in order to translate this approach to humans and veterinary applications. ${ }^{27}$

Based on this context, we constructed DNA vaccines encoding the RVFV eGn in fusion with a scFv anti-ovine DEC205 and a scFv anti-ovine CD11c and compared them to a DNA vaccine encoding untargeted $\mathrm{eGn}$ for their capacity to induce protective immunity in sheep. The vaccines were delivered in the dermis, a tissue densely inhabited with DCs, together with an adjuvant plasmid encoding for ovine GM-CSF. ${ }^{28}$ Surface electroporation (SEP) was used to promote DNA transfection in skin cells and immunogenicity. $^{29}$ Interestingly, we found that the DNA encoding eGn targeted to DEC205 and not to CD11c promoted the interferon$Y$ (IFN $\gamma$ ) T-cell responses as compared to DNA encoding untargeted eGn, thus indicating that APC targeting can improve T-cell responses outside the mouse species. However, DNA encoding untargeted eGn was the most efficient at inducing anti-eGn immunoglobulin $\mathrm{G}$ ( $\mathrm{lgG})$ responses, although not neutralizing. This latter plasmid was efficient at inducing significant protection against infectious challenge to a higher extent than the one encoding DEC205- and CD11c-targeted eGn. Furthermore, the anti-eGn lgG levels correlated with protection, suggesting that $\lg G$ responses rather than T-cell responses are important in the protective immunity conferred by eGn encoding DNA vaccines.

\section{RESULTS}

Generation and validation of scFvs for targeting RVFV eGn to ovine DEC205 and CD11c receptors

In order to construct DNA vaccines encoding RVFV eGn targeted to sheep APCs, we derived ScFv sequences from two murine hybridomas secreting monoclonal antibodies (mAbs) against ovine DEC205 or CD11c. The SCFv sequences were fused to mCherry and the fusion proteins were expressed in HEK293 cells. We evaluated the binding capacities to sheep CDC subsets of scDEC-mCherry and scCD11c-mCherry fusion proteins relatively to a scCtIr-mCherry control, and we compared their staining profiles to the ones of their parental mAb. For that purpose, we collected lymph-draining sheep skin at steady state using the pseudoafferent lymph cannulation technique ${ }^{23}$ and we isolated lowdensity cells; these cells are strongly enriched in skin-migrated cDCs which are physiologically relevant cell targets in the context of skin vaccination and which include the $\mathrm{CDC1}$ and CDC2 subtypes, identified as $\mathrm{FSC}^{\text {hi }} \mathrm{CD} 1 \mathrm{~b}^{+} \mathrm{CADM}^{\mathrm{hi}}$ and $\mathrm{FSC}^{\text {hi }}$ $\mathrm{CD} 1 \mathrm{~b}^{+} \mathrm{CADM} 1^{\text {lo }}$ cells respectively (Fig. $1 \mathrm{a}$ ). ${ }^{20,23} \mathrm{~A}$ staining with the parental anti-DEC205 mAb shows that CDC1s express slightly more DEC205 than CDC2s, relatively to an isotype control (Fig. 1b), as we previously published. ${ }^{23}$ This finding is in agreement with the slightly higher expression of DEC205 on mouse skin-migrated $\mathrm{CDC} 1 \mathrm{~s}$ than on $\mathrm{CDC} 2 \mathrm{~s} .{ }^{30}$ The staining of $\mathrm{CDC} 1 \mathrm{~s}$ with the parental anti-CD11c mAb appears heterogeneous and presented two peaks, whereas the staining of CDC2 is homogenous. The scDECmCherry and scCD11c-mCherry fusions presented a similar staining pattern as the one obtained with the parental mAbs (Fig. 1c). Thus, the anti-DEC205 and CD11c scFvs can be used to create constructs to target RVFV eGn to the corresponding sheep molecules.

Construction, characterization and expression of anti-DEC205 and anti-CD11c scFvs fused to RVFV eGn

Synthetic genes were designed in order to generate vaccine plasmids encoding untargeted eGn (peGn) and eGn targeted to DEC205 or to CD11c using the validated scFv sequences (pscDECeGn and pscCD11c-eGn). As shown in Fig. 2a, peGn includes the eGn sequence (nt 411 to nt 1763 of the RVFV M segment, ZH501 strain) beginning with its natural signal peptide (nt 411 to 477) whereas the plasmids encoding APC-targeted eGn include the $\mathrm{VH}$ signal peptide of the original $\mathrm{mAb}$, the scFv sequence and the eGn sequence from nt 477 to nt 1763 which is devoid of transmembrane domains for allowing the fusion protein secretion.

The plasmids were transfected in HEK293 cells and the cell lysates and supernatants were collected for assessing eGn protein expression in vitro. Bands corresponding to untargeted eGn $(48.8 \mathrm{kDa})$ and to scDEC-eGn and scCD11c-eGn protein fusions (76.3 and $78.6 \mathrm{kDa}$ respectively) were detected at their expected predicted size in western blot using an anti-RVFV hyperimmune mouse ascitic fluid (HMAF, Fig. 2b). We could not detect the three proteins in the concentrated supernatants by western blot, possibly due to too limited amounts of eGn that could be detectable by the anti-RVFV HMAF on the nitrocellulose membrane. However, in a dot plot assay where 25 times more volume was loaded, the three supernatants from the eGn-expressing plasmid reacted with the anti-RVFV HMAF (Fig. 2c). Furthermore, when low-density skin lymph cells were incubated with the concentrated supernatants, the binding of scDEC-eGn and scCD11c-eGn could clearly be detected by the anti-RVFV HMAF on FSC hi cells, which mainly correspond to CDCs (Fig. 2d). This experiment indicates that our scFv-based construct can target eGn to sheep skin DCs.

Finally, we assessed whether peGn, pscDEC-eGn and pscCD11ceGn can be expressed in vivo, and to what relative levels, in the sheep host species. We chose to deliver the plasmids into sheep skin by injection in the dermis followed by SEP, as done previously to successfully immunize mice and pigs with DNA encoding for APC-targeted vaccines. ${ }^{15,16}$ After having determined the optimal plasmid delivery parameters using a firefly luciferase expressing plasmid and escalating electric field values (supplementary material 1), we detected the expression of peGn, pscDEC-eGn and pscCD11c-eGn delivered in sheep skin, using the sensitive real-time reverse transcription-polymerase chain reaction (qRT$P C R$ ) technique, $48 \mathrm{~h}$ post administration (Fig. 2e). eGn messenger RNA (mRNA) could be detected in all injected biopsies (from Cq 26 to $\mathrm{Cq} 32.8$, data not shown) and no signal could be detected in the non-transfected skin. After normalization with the RPS24 reference gene, we found that eGn mRNA expression was quite variable for 


\section{A Gating strategy}

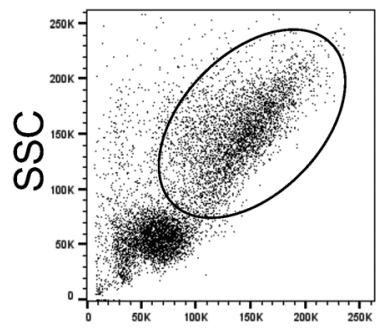

FSC

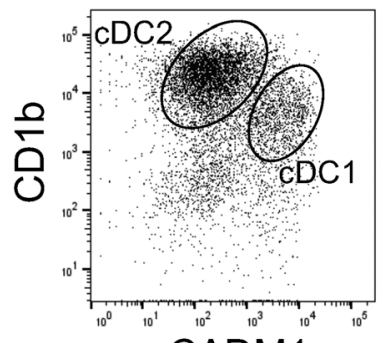

CADM1

\section{B mAb}
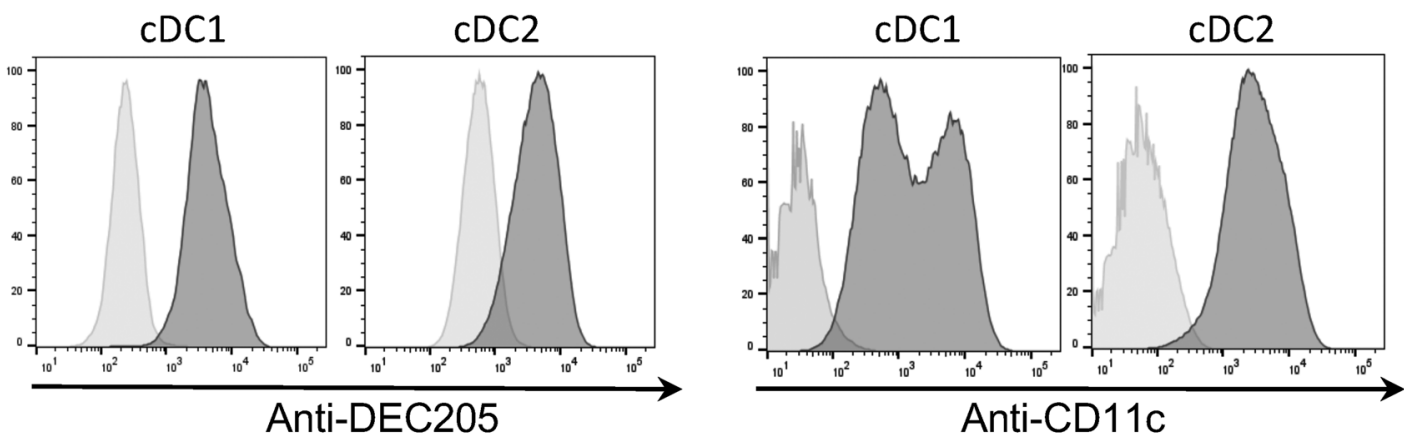

\section{ScFv}
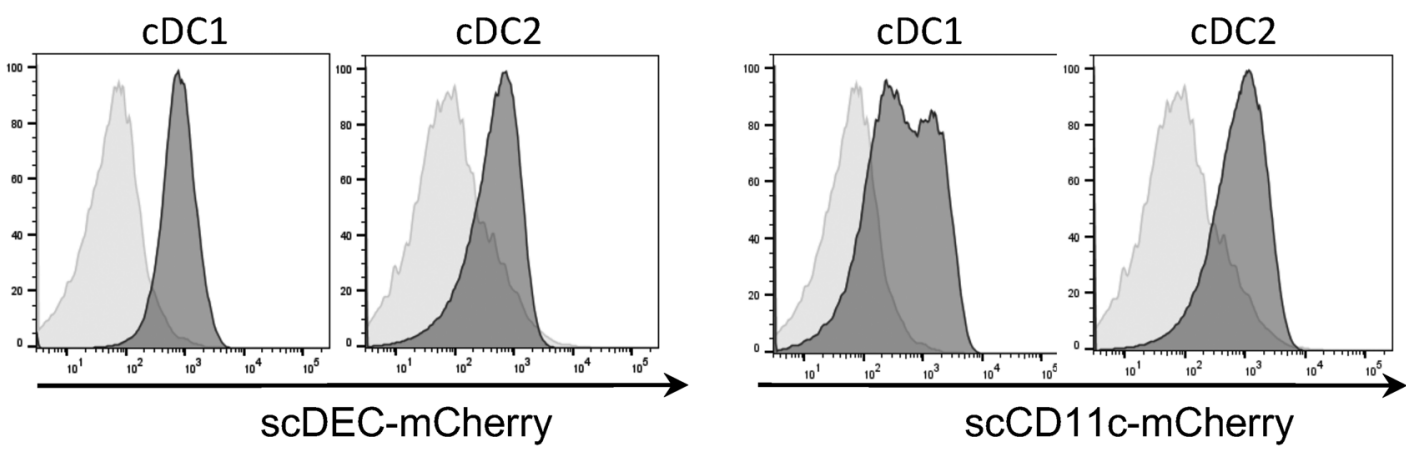

Fig. 1 Binding of anti-DEC205 mAb, anti-CD11c mAb, scCD11c-mCherry and scDEC-mCherry on sheep skin lymph DC subsets. a Sheep skin lymph low-density cells were gated to select FSC ${ }^{\text {hi }}$ cells which were further separated as CD $1 b^{+}$CADM $1^{\text {hi }}$ and $C D 1 b^{+} C A D M 1{ }^{10}$ cells, corresponding mainly to $\mathrm{CDC1s}$ and $\mathrm{CDC} 2 \mathrm{~s}$ respectively. $\mathbf{b}$ The staining of the $\mathrm{CDC1}$ and $\mathrm{CDC} 2$ subsets with the parental anti-DEC205 and antiCD11c mAbs is depicted (dark gray) compared to an isotype control (pale gray). c The staining of the CDC1 and CDC2 subsets with scDEC$m$ Cherry and scCD11c-mCherry is depicted (dark gray) compared to a scCtrl-mCherry (pale gray). The shown data are representative of two independent experiments

the same plasmid, precluding any statistical analysis. Nevertheless, pscDEC and pscCD11c-eGn tended to be less expressed or were at least not more expressed than peGn in sheep skin (Fig. 2e).

In conclusion, pscDEC-eGn and pscCD11c-eGn express functional fusion proteins capable of targeting eGn to sheep APCs and the mRNA expression of peGn, pscDEC-eGn and pscCD11c-eGn is detectable in pig skin upon electroporation in vivo.

Immunization with pscDEC-eGn promotes the eGn-specific IFNY T-cell responses

The three RVFV DNA vaccines, i.e., peGn, pscDEC-eGn and pscCD11c-eGn, were compared for their immunogenicity in sheep. Each DNA vaccine was combined to pGM-CSF as an adjuvant and was inoculated 3 times intradermally+SEP in 2-month-old Lacaune lambs (Fig. 3a). At week 12 after the prime, peripheral blood mononuclear cells (PBMCs) were re-stimulated in vitro with two pools of overlapping peptides covering the entire eGn sequence (a N-ter and a C-ter peptide pool) in an IFNy EnzymeLinked ImmunoSpot (ELISPOT) assay. Cultures with an irrelevant peptide were used as controls (see Materials and methods section). eGn-specific IFNy responses were observed upon restimulation with both the eGn N-ter and C-ter pool peptides in the all-vaccinated groups versus the control sheep groups (Fig. 3b). The most significant difference of response versus the control group was obtained with the cells from the pscDEC-eGn vaccinated sheep $(p<0.01$ with the $\mathrm{N}$-ter pool and $p=0.05$ with the C-ter pool). The IFNy spot number induced by the eGn N-ter pool was significantly higher with the PBMCs from the pscDECeGn- than from the peGn vaccinated sheep (Fig. $3 b$ ). Although not more expressed than peGn at the RNA level in sheep skin, pscDECeGn appears more potent than pscCD11c-eGn and peGn to induce IFNY T-cell responses. 
A
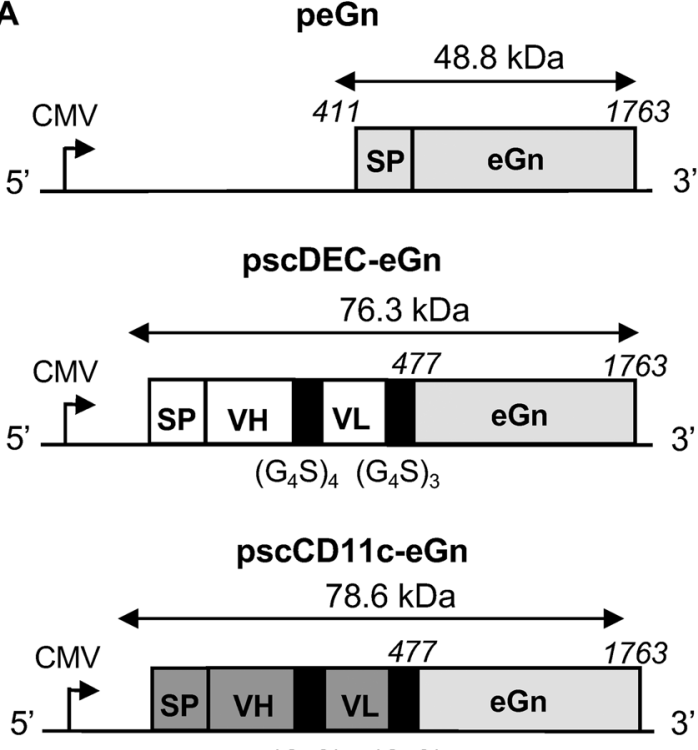

$\left(\mathrm{G}_{4} \mathrm{~S}\right)_{4}\left(\mathrm{G}_{4} \mathrm{~S}\right)_{3}$
B

$\begin{array}{lllll}M W & 1 & 2 & 3 & 4\end{array}$

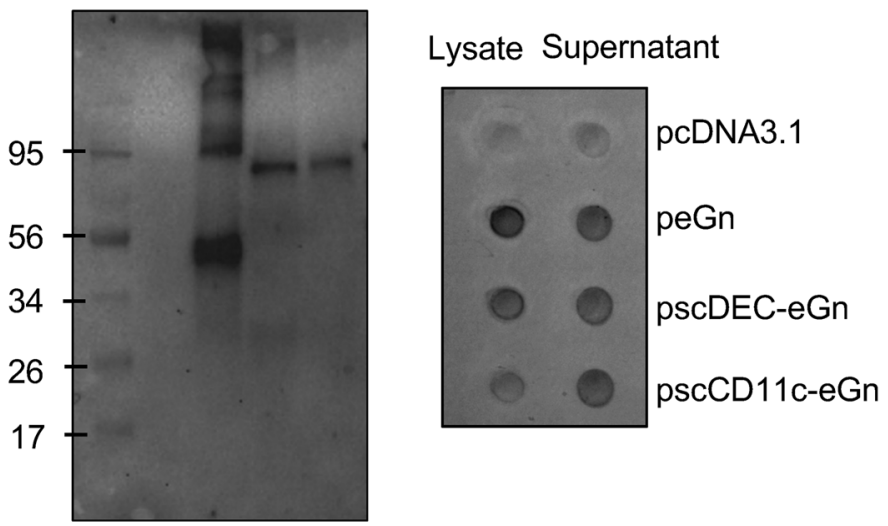

1: pcDNA3.1

2: peGn

3: pscDEC-eGn

4: pscCD11c-eGn
C

E

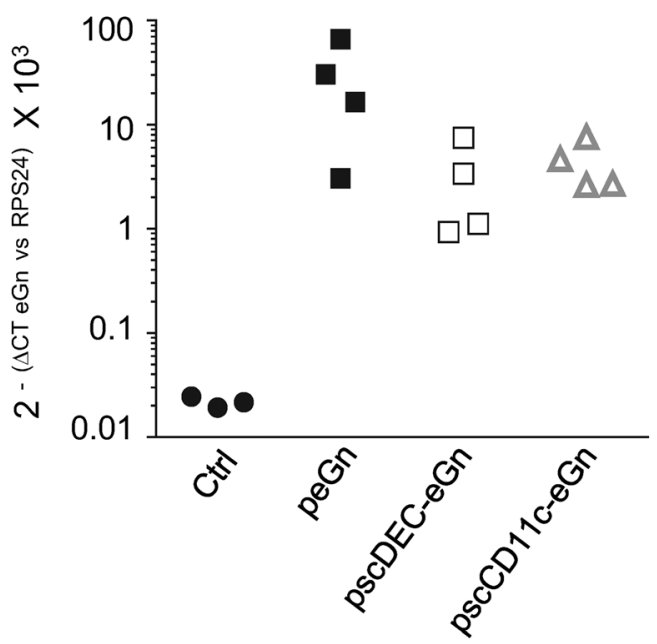

Fig. 2 Design and expression of peGn, pscDEC-eGn and pscCD11c-eGn. a Schematic representation of peGn, pscDEC-eGn and pscCD11c-eGn. peGn includes the codon-optimized eGn sequence with its intrinsic signal peptide (SP, RVFV M segment from nt 411 to nt 1763 ) and cloned in the pcDNA4-V5His expression vector. pscDEC-eGn and pscCD11c include the signal peptide (SP) of the VH chain of the parental mAb sequence, the scFv (VH and VL) sequence and the nt 477 to nt 1763 portion of the codon-optimized eGn sequence. The VH and VL sequences are connected together with a $\left(\mathrm{G}_{4} \mathrm{~S}\right)_{4}$ linker and the $\mathrm{scFv}$ and $\mathrm{eGn}$ sequences are connected with a $\left(\mathrm{G}_{4} \mathrm{~S}\right)_{3}$ linker. These chimeric sequences were cloned in a pcDNA3.1 vector. b, c HEK293 cells were transfected with pcDNA3.1, peGn, pscDEC-eGn and pscCD11c-eGn. In (b), the cell lysates $(10 \mu \mathrm{l})$ were separated by SDS-PAGE under reducing conditions and eGn was revealed with anti-RVFV HMAF followed by a HRP-GAM IgG. The predicted sizes of the expressed untargeted and chimeric eGn proteins are $48.8 \mathrm{kDa}$ for untargeted eGn, $76.3 \mathrm{kDa}$ for the scDEC-eGn protein and $78.6 \mathrm{kDa}$ for the scCD11c-eGn protein. All samples were gathered from the same experiment and were processed in parallel (1 repeated experiment). In (c) the cell lysates $(100 \mu \mathrm{l})$ and the concentrated cell supernatants $(500 \mu \mathrm{l})$ gathered from the same experiment were spotted onto the same nitrocellulose membrane and the eGn protein was revealed as in (b) (1 repeated experiment). $\mathbf{d}$ Sheep skin lymph lowdensity cells were reacted with the parental anti-DEC205, anti-CD11c or isotype control mAbs (top panel) or with the concentrated supernatant from HEK293 cells transfected with peGn, psCDEC-eGn, pscCD11c-eGn and a negative control pcDNA3.1 (bottom panel). The staining of the DAPI ${ }^{\text {neg }} \mathrm{FSC}^{\text {hi }}$ cells, which include dominantly CDCs, is depicted (dark gray) compared to control (dash line). Bound mAbs were revealed with A488-DAM IgG and bound eGn with anti-RVFV HMAF followed by A488-DAM IgG. e Relative expression of peGn, pscDEC-eGn and pscCD11c-eGn in sheep skin. peGn, psCDEC-eGn and pscCD11c-eGn (100 $\mu \mathrm{g})$ were injected intradermally in $100 \mu \mathrm{l}$ saline in inner front leg sites of two sheep followed by SEP $(532 \mathrm{~V} / \mathrm{cm})$. After $48 \mathrm{~h}$, RNA was extracted from skin biopsies. eGn mRNA levels were measured using qRTPCR, normalized with RPS24 ribosomal RNA and expressed as $2^{-\Delta C T}$ values (1 repeated experiment). Absence of residual plasmid in the RNA preparations was controlled using $\mathrm{qPCR}$ without reverse transcription 
A
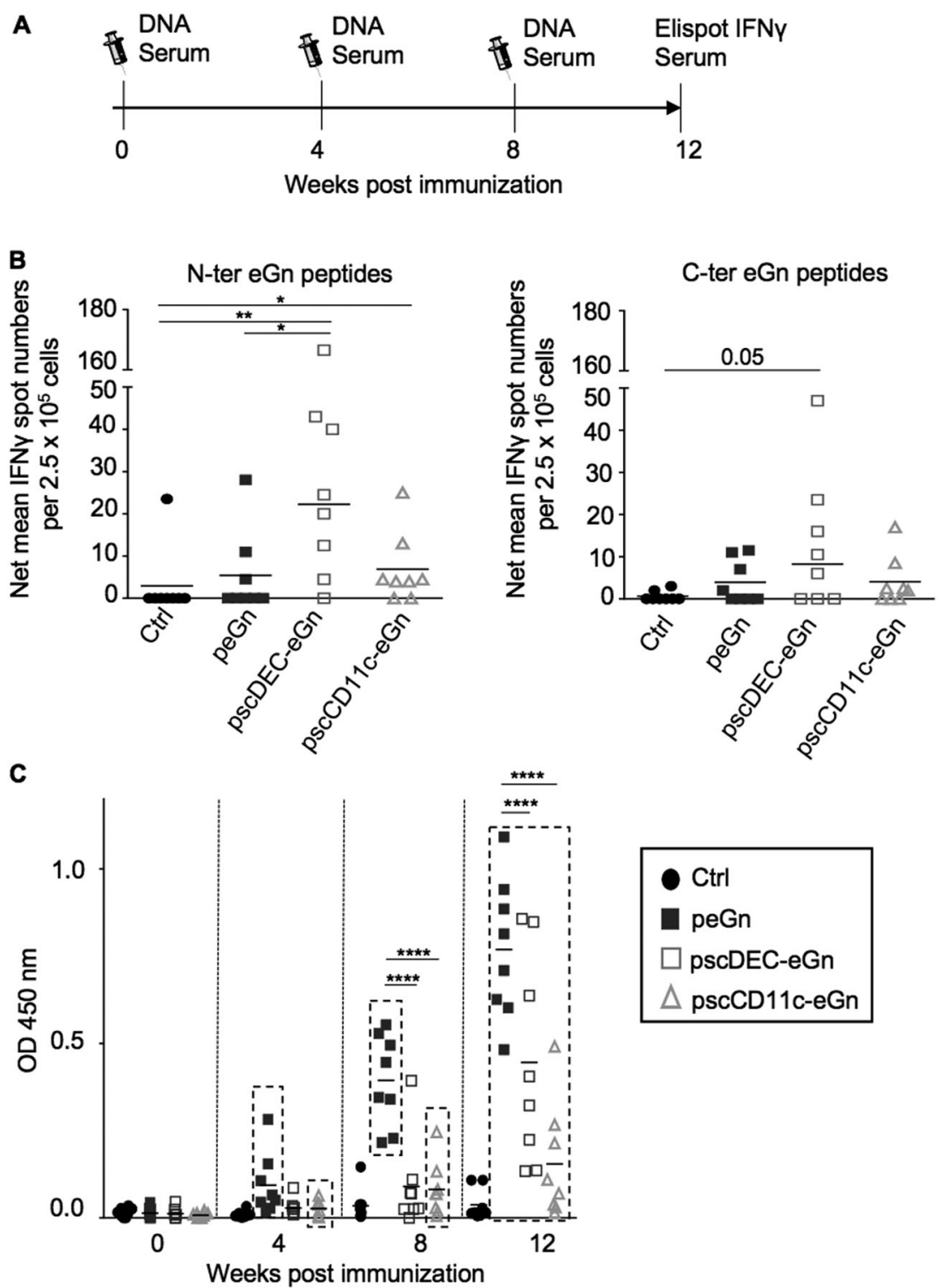

Fig. 3 IFN $\gamma$ T-cell and $A b$ responses induced by peGn, pscDEC-eGn and pscCD11c-eGn in lambs. a Schematic representation of the immunization protocol. The 2-month-old lambs were immunized at weeks 0,4 and 8 with $400 \mu \mathrm{g}$ of peGn, pscDEC-eGn, pscCD11c-eGn and $400 \mu \mathrm{g}$ of pGM-CSF as adjuvant. Control lambs received pGM-CSF only. The plasmids were injected intradermally and the injection was immediately followed by SEP $(532 \mathrm{~V} / \mathrm{cm})$. Sera were collected and the IFN $\gamma$ T-cell responses were assessed by ELISPOT at the indicated time points. The syringe drawing is from Servier Medical Art which provides open source illustrations. b PBMCs $\left(2.5 \times 10^{5}\right)$ were plated in Multiscreen plates coated with a capture anti-ovine IFN $\gamma$ mAb (duplicated wells) and re-stimulated for $18 \mathrm{~h}$ with overlapping eGn peptides covering the $\mathrm{N}$ - and C-terminal parts $(5 \mu \mathrm{g} / \mathrm{ml})$, or with an irrelevant peptide $(5 \mu \mathrm{g} / \mathrm{ml})$. IFN $\gamma$ spot counts were considered eGn specific when both duplicated well values from cells stimulated with eGn peptides were strictly superior to the duplicated well values from cells stimulated with the irrelevant peptide. The mean spot numbers in stimulated wells minus the ones stimulated with irrelevant peptides are shown (net mean values). Each symbol in the graphs represents an individual animal and the mean is indicated. $P$ values between the two groups were determined according to the Mann-Whitney test $\left({ }^{*} p<0.05 ;{ }^{* *} p<0.01\right)$. c The serum of the lambs immunized with peGn, pscDEC-eGn, pscCD11c-eGn and of control lambs were assayed for the detection of eGn-specific lgG using indirect ELISA. A lysate from HEK293 cells transfected with peGn was used as coating (see Materials and methods section) and the individual sera were tested in a 1:100 dilution. The OD signals at $450 \mathrm{~nm}$ from the sera collected at weeks $0,4,8$ and 12 are shown in $3 C$ ( 1 repeated experiment). Each lamb serum is shown as a distinct symbol and the mean OD value is represented as an horizontal bar. $P$ values were determined using the two-way ANOVA with Bonferroni's correction to evaluate the statistical significance of the OD value differences between vaccinated groups ${ }^{*} p<0.05$; ${ }^{* *} p<0.01$; $\left.{ }^{* * * *} p<0.0001\right)$ and the significance of the differences between the OD values measured at the different weeks versus at day 0 was evaluated for each vaccinated group using paired $t$-test and the groups with statistically significant differences are indicated by a box

Immunization with peGn promotes the anti-eGn IgG responses in sheep sera

Anti-eGn lgGs were measured in indirect enzyme-linked immunosorbent assay (ELISA). The OD450 nm results from 1:100 diluted sera are reported in Fig. 3c. Significant detection of anti-eGn IgG levels was obtained after one injection in the peGn and pscCD11ceGn-vaccinated group ( $p<0.05$ in comparisons versus week 0 , Fig. $3 c$ ). The induction of significant anti-eGn lgG levels was reached for all groups after three inoculations (Fig. 3c). The levels of antieGn lgG were higher in the sera from peGn- versus pscCD11c-eGn- 
and pscDEC-eGn-vaccinated sheep at weeks 8 and 12, and they tended to be higher in the pscDEC-eGn- than in the pscCD11ceGn-vaccinated sheep at week 12 (Fig. 3c). End-point titers were calculated from serially diluted sera and gave similar differences between groups (Supplementary material 3). The ELISA-positive sera were tested for their capacity to neutralize RVFV in a reduction plaque assay. None of the sera were neutralizing at the difference with positive control sera from infected sheep (data not shown). Overall, there was a graded capacity of our RVFV DNA vaccines for induction of anti-eGn IgG, with peGn being the most potent and pscCD11c-eGn being least potent, and the anti-eGn IgGs were not capable of neutralizing RVFV in vitro.

peGn and to a lesser extent pscDEC-eGn induce partial protection against infectious RVFV challenge in sheep

Sheep were challenged at week 12 with the RVFV 56/74 strain, a previously demonstrated virulent strain in sheep. ${ }^{31}$ The infected sheep were monitored for clinical symptoms and temperature, and sera were collected regularly for viral RNA and infectious particle detection (see Materials and methods). Two sheep died in the control group at 6 and 8 days post challenge (dpc), and none in the vaccinated groups (Table 1). The post-mortem lesions included severe pulmonary edema, hemorrhages in multiple organs, generalized lymphadenopathy and multifocal liver necrosis. The mean body temperature reached a peak over $41^{\circ} \mathrm{C}$ in the control, pscCD11c-eGn and pscDEC-eGn vaccinated groups (41.06, 41.23 and $41.33^{\circ} \mathrm{C}$ respectively), whereas the mean body temperature was significantly reduced to $40.05^{\circ} \mathrm{C}$ in the peGn vaccinated sheep $(p<0.0001)$ (Fig. $4 a)$. The total clinical score (Table 1), reflecting the severity of the disease (see supplementary material 2 for scoring), was the highest in the control group (92), slightly reduced in the pscCD11c-eGn group (44) and much decreased in the pscDEC-eGn (29) and peGn (22) vaccinated group (Table 1). The main symptoms were nasal mucus discharges, decreased activity, anorexia and conjunctive redness. The viral RNA copies per $\mathrm{ml}$ serum were measured on 1, 2, 3, 4, 5, 6,8 and $10 \mathrm{dpc}$ for each sheep, except for the two dead sheep in which copies were monitored up to $6 \mathrm{dpc}$ (Fig. 4b). The mean integrated value of the viral RNA copies per ml serum (viral RNA area under the curve (AUC), see Materials and methods) reached $3.87 \times 10^{12}$ in the control sheep and was significantly decreased in the pscDEC-eGn and peGn vaccinated sheep $\left(6.39 \times 10^{10}, p<0.05\right.$ and $4.41 \times 10^{9}, p<0.01$ respectively, Fig. $4 \mathrm{c}$ ). The viral RNA AUC mean value of the pscCD11c-eGn group was slightly but not significantly reduced compared to the one of the control group $\left(3.89 \times 10^{11}\right)$. The peGn vaccinated sheep cleared the viral RNA copies in serum on $6 \mathrm{dpc}$, the pscDEC-eGn vaccinated sheep on 8 $\mathrm{dpc}$ and the pscCD11c-eGn vaccinated and control group on 10 $\mathrm{dpc}$. The particle-forming units (pfu) per $\mathrm{ml}$ serum were measured over 8 days (Fig. 4d); the mean integrated values (pfu AUC, see Materials and methods) were calculated and followed the same trend as the viral RNA ones (Fig. 4e). The peGn vaccinated sheep cleared the infectious particles in serum on $5 \mathrm{dpc}$, the pscDEC-eGn vaccinated sheep and the pscCD11c-eGn vaccinated on $4 \mathrm{dpc}$ and control group on $8 \mathrm{dpc}$. Finally, the IgG responses directed to eGn, to total RVFV antigens, and to $\mathrm{N}$ antigens and the neutralizing Abs (NAbs) were monitored following infection (supplementary material 4). The anti-eGn lgG response was boosted between 5 and $6 \mathrm{dpc}$ in the vaccinated groups, reached the highest levels in the peGn vaccinated sheep $(p<0.05)$, whereas the control sheep developed detectable responses only from $10 \mathrm{dpc}$, reaching much lower levels than in the vaccinated sheep. The anti-RVFV total antigen IgG followed a similar trend. The titers of the NAbs at 10 $\mathrm{dpc}$ reached similar levels in all groups (supplementary material 4), indicating that the DNA vaccines did not induce a clear priming of NAbs, whereas they clearly primed the anti-eGn and anti-RVFV IgG responses which were boosted by the viral challenge. Notably, in
Table 1. Clinical scores upon infectious challenge in vaccinated lambs

\begin{tabular}{llll}
\hline & Individual scores $^{\mathrm{a}}$ & $\begin{array}{l}\text { Total group } \\
\text { score }^{\mathrm{b}}\end{array}$ & $\begin{array}{l}\text { Death per } \\
\text { group }\end{array}$ \\
\hline Control & $21,6,2,13,39,1,1,9$ & 92 & $2 / 8$ \\
peGn & $4,2,4,2,0,8,2,0$ & 22 & $0 / 8$ \\
psCDEC-eGn & $0,11,4,2,7,3,2,0$ & 29 & $0 / 8$ \\
pscCD11c-eGn & $3,8,6,5,5,7,8,1$ & 43 & $0 / 8$ \\
\hline
\end{tabular}

The individual score was calculated by adding the daily clinical score over 20 days. The daily clinical score was established by monitoring anorexia, eye aspect, activity/movement, lack of rumination, nasal mucus, prostration, respiratory movement, tremor ( 1 to 3 was attributed depending of severity) and death ( 15 was arbitrarily attributed)

${ }^{\mathrm{b}}$ The total group score was calculated by adding the individual scores of the group

agreement with an efficient control of infection by the DNA vaccines, the IgG response against the $\mathrm{N}$ immunodominant viral protein, to which all sheep were naive at the time of challenge, was lower in the vaccinated groups than in the control group $(p<$ 0.05 at $10 \mathrm{dpc}$ ).

So far, our data show that peGn was efficient at inducing significant levels of protective immunity, reducing by about 3 log10 the RNA viral loads and accelerating viral clearance, in association with a strong decrease in the clinical symptoms severity. pscDEC-eGn, which induced higher T-cell responses and lower anti-eGn lgG responses than eGn, revealed to be less protective than peGn, suggesting that the IFNy T-cell response is less critical than the $A b$ response in the protective immunity induced by these vaccines. Finally, pscCD11c-eGn, which was the least efficient at triggering anti-eGn lgG responses, did not induce significant protection.

The anti-eGn IgG levels at challenge correlate with virological and clinical protection

In order to get further insight into the immune parameter(s) associated with protection against RVFV induced by our DNA vaccines, we took advantage of the fact that the three DNA vaccines induced a relatively wide range of values of immune, virological and clinical parameters. These parameter values were loaded in a principal component analysis (PCA) in order to identify the main axes of the data variance as well as the key variables. In a first analysis where we used the data of the control and vaccinated groups, the PC1 axis, which explains $36.82 \%$ of the variance, clearly separates the data from the control and the vaccinated group, with the peGn vaccinated group data being the most distant from the control group data (Fig. 5a). In a second analysis using the vaccinated group data, the PCA loading from each parameter shows that the clinical scores, the viral RNA and the pfu cluster together and oppose the anti-eGn and anti-RVFV IgG levels at $0 \mathrm{dpc}$ and less so at 6 and later dpc (Fig. 5b). The T-cell responses, materialized by a short arrow, do not generate any significant pattern (Fig. 5c). Finally, we performed one to one correlation analyses (Spearman's test) and found that the best negative correlation index was obtained between the pfu AUC and the anti-eGn IgG levels at $0 \mathrm{dpc}(r=-0.6, p<0.01$, Fig. $5 c$ and supplementary material 5), and the correlation significance was lost at later time points. A similar negative correlation was obtained between the clinical scores and the anti-eGn lgG levels. No correlation could be found between the T-cell responses and the virological or clinical parameters.

Altogether, the global analysis of the immune, virological and clinical data of sheep vaccinated with peGn, pscDEC-eGn and pscCD11c-eGn indicate that anti-eGn lgG levels at the time of challenge are associated with protection and suggest that these 
A

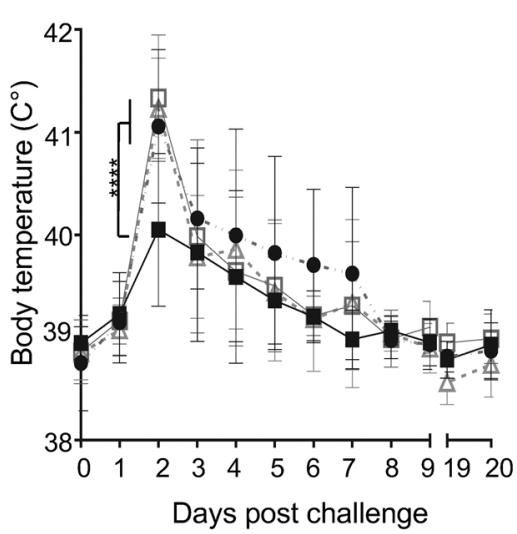

B

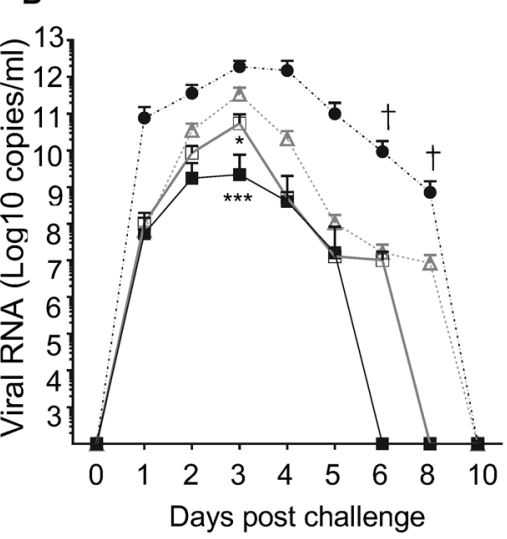

C

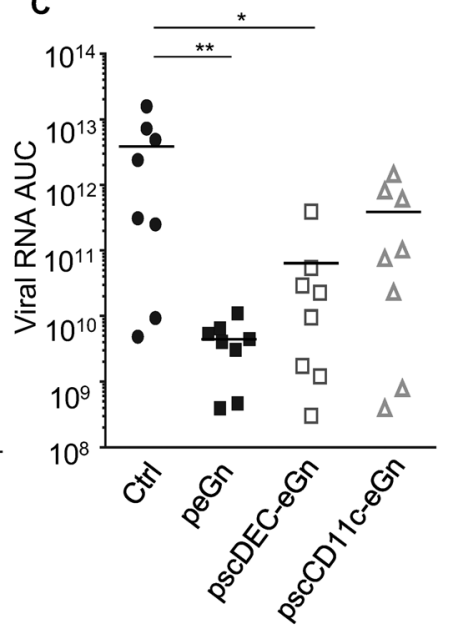

D

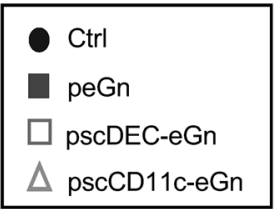

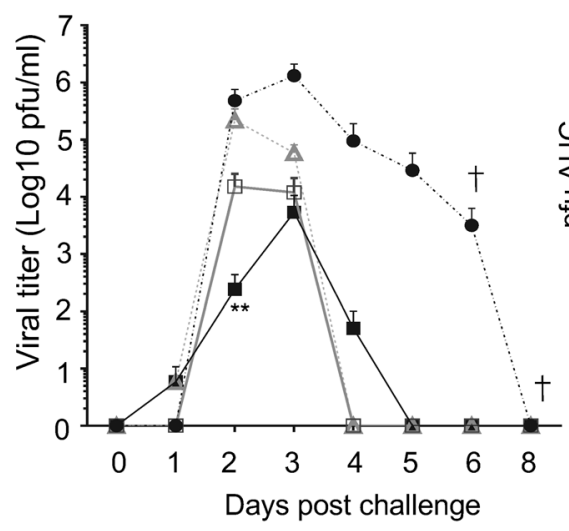

E

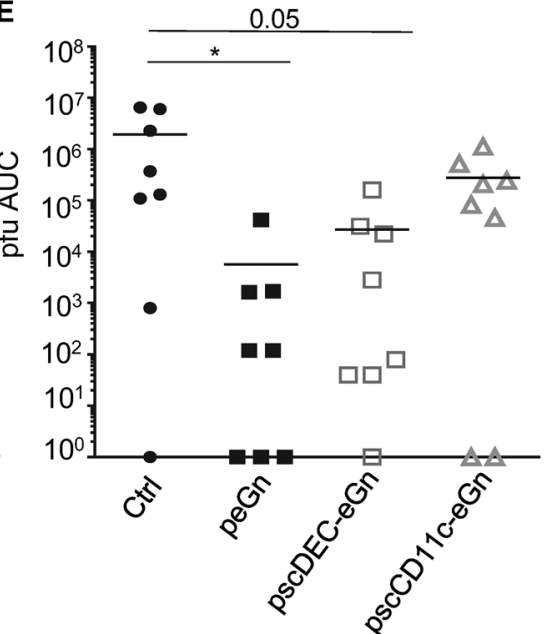

Fig. 4 Body temperature and viremia upon RVFV challenge in lambs vaccinated with peGn, pscDEC-eGn and pscCD11c-eGn. The vaccinated and control sheep were challenged with the RVFV $56 / 74$ strain $\left(10^{6} \mathrm{TCID}_{50}\right) 3$ weeks after the last immunization. a The rectal temperature was measured daily. Data are mean \pm SD of individual values $(n=8$ sheep per experimental group). The $p$ values were determined according to a two-way ANOVA test with Bonferroni's correction (****p $<0.0001)$. b The viral RNA copy numbers/ml in serum were quantified by qRT-PCR and the mean values for each group with the top standard error of mean are shown over times. c The viral RNA area under the curve (viral RNA AUC) was calculated over 10 days for each animal. d The plaque-forming unit load (pfu/ml) were quantified in viral RNA-positive sera and the mean values for each group with the top standard error of mean are shown over times. e The pfu area under the curve (pfu AUC) was calculated over 8 days. Each symbol represents an individual animal and the mean is indicated by a horizontal line $(n=8$ per experimental group). In (b-d), the $p$ values between the vaccinated and control groups were determined with the Mann-Whitney test $\left({ }^{*} p<0.05 ;{ }^{* *} p<0.01\right.$; $\left.{ }^{* * *} p<0.001\right)$. A cross symbol indicates death

Abs, although not neutralizing in plaque assay, were instrumental in the protective immunity induced by our DNA vaccines.

\section{DISCUSSION}

In this work, we showed that a DNA vaccine encoding untargeted eGn conferred significant protection against a RVFV challenge in sheep. Our finding suggested that the anti-RVFV protective immunity relied on antibodies, although not neutralizing, and not on IFNy-producing T cells. However, polyfunctional cytokine secretion by $T$ cells and cytotoxicity, which were not assessed here, could also play a role. Importantly, our results indicate that targeting antigens to DEC205 can be used to improve the T-cell response in ruminants when this type of response would be beneficial.

The formalin-inactivated and live-attenuated vaccines have been licensed in African countries where RVFV is endemic (see ref. 32 for recent review on RVFV vaccines). However, inactivated vaccines require a booster and annual revaccinations, the live- attenuated Smithburn vaccine is teratogenic and the liveattenuated clone 13 , which has a higher safety profile associated to a large deletion in the small segment, can nevertheless induce abortion during the first trimester of gestation. ${ }^{33}$ With the goal to improve safety, next-generation live-attenuated vaccines, such as a reassortant between clone 13 and the MP-12 chemically attenuated strain $^{34}$ or MP-12-derived clone with silent mutations, ${ }^{35}$ have been developed. However, there is resistance of many countries to authorize live-attenuated vaccines, due to the risk of reversion to virulence. Therefore, other vaccine candidates were generated and demonstrated promising results in sheep and include subunit vaccines, ${ }^{36,37}$ virus-like particles, ${ }^{36}$ virus replicon particle vaccines, ${ }^{34}$ virus-vectored vaccines ${ }^{36,38,39}$ and DNA. ${ }^{40}$ In contrast to our results, a DNA vaccine encoding for the glycoprotein precursor Nsm/Gc/Gn did not induce T- or B-cell response in sheep, using 3 injections of $400 \mu \mathrm{g}$ DNA in lipofectin as a delivery method. ${ }^{40}$ Several of these novel candidates were compared to a commercial vaccine, either to an inactivated vaccine $^{36}$ or to clone $13 .^{34}$ In the first study, the inactivated 
A

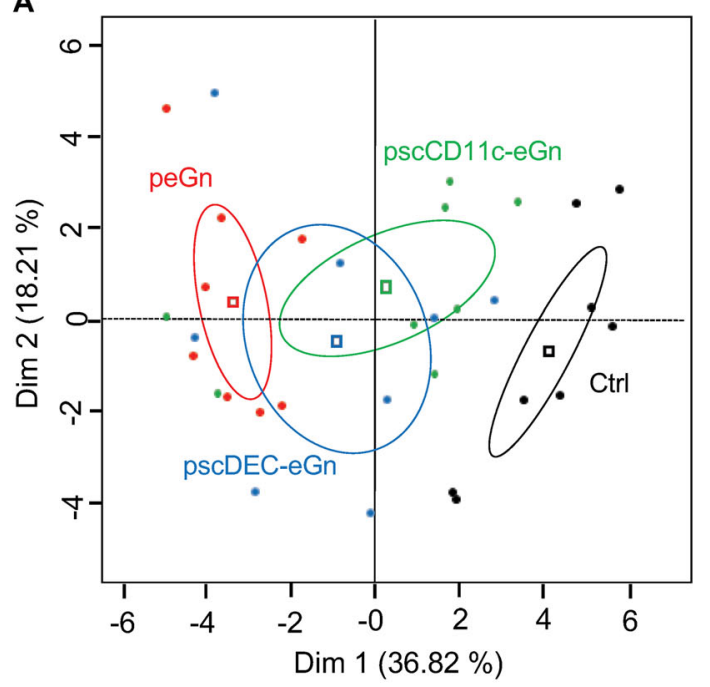

B

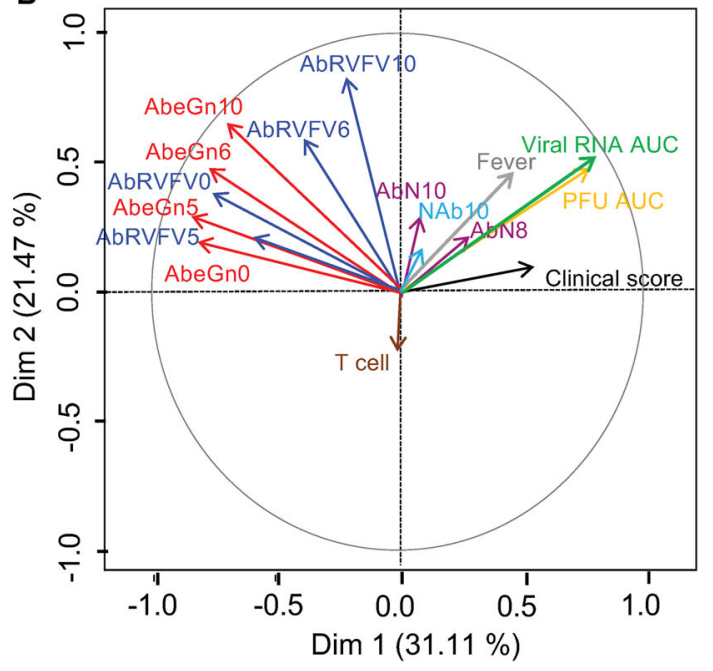

C

\begin{tabular}{cccc}
\cline { 2 - 4 } dpc & 0 & 5 & 6 \\
Clinical score & $-0.49^{*}$ & -0.37 & -0.31 \\
Viral RNA AUC & $-0.55^{\star *}$ & $-0.46^{*}$ & -0.36 \\
pfu AUC & $-0.60^{* *}$ & $-0.50^{*}$ & -0.36 \\
Spearman r & & & \\
& -0.6 & -0.4 &
\end{tabular}

\begin{tabular}{ccc}
\multicolumn{3}{c}{ Anti-RVFV } \\
\hline 0 & 5 & 6 \\
$-0.50^{*}$ & -0.32 & 0.05 \\
$-0.44^{*}$ & -0.37 & -0.15 \\
$-0.52^{* *}$ & $-0.48^{*}$ & -0.12 \\
& & \\
\end{tabular}

\begin{tabular}{|c|c|c|c|c|}
\hline \multicolumn{3}{|c|}{ Anti-N } & $\mathrm{T}$ cell & NAbs \\
\hline 5 & 6 & 10 & 0 & 10 \\
\hline-0.30 & -0.11 & -0.19 & 0.00 & 0,15 \\
\hline-0.17 & -0.02 & 0.24 & -0.12 & 0,28 \\
\hline-0.18 & -0.08 & 0.27 & -0.13 & 0,27 \\
\hline
\end{tabular}

0,27

Fig. 5 PCA and correlation analysis of the clinical, virological and immune response data of the lambs vaccinated with peGn, pscDEC-eGn and pscCD11c-eGn. a A PCA plot of the clinical, virological and immune response data (=PCA variables) of vaccinated and non-vaccinated sheep is depicted, with each sheep represented as a dot in a specific color according to its group assignment: PC1 explains $36.82 \%$ of the total variation of the data between sheep and PC2 explained a further $18.21 \%$ of the variation. The vaccination regimen is indicated by a distinct color (red for peGn, blue for pscDEC-eGn, green for pscCD11c-eGn). b Thirty-one individual input variables were loaded (see Materials and methods) and the most relevant ones are indicated on the figure for clarity: viral RNA AUC, pfu AUC, individual clinical scores, integrated temperature measurements, T-cell responses at week 12, anti-eGn lgG OD signals (AbeGn) at 0, 5, 6 and $10 \mathrm{dpc}$, anti-N lgG OD signals (AbN) at 8 and $10 \mathrm{dpc}$, NAb titers (NAb) at $10 \mathrm{dpc}$ and anti-RVFV IgG OD signals (AbRVFV) at 0, 5, 6 and 10 dpc. c Correlation analyses between the immune response data versus the clinical and virological data. For each vaccinated lamb, the anti-eGn and anti-RVFV IgG OD signals at 0,5 and $6 \mathrm{dpc}$, the NAb titers at $10 \mathrm{dpc}$ and the anti-N IgG OD signals at 5, 6 and $10 \mathrm{dpc}$ were subjected to bilateral Spearman's correlation analysis against the individual clinical scores, the viral RNA AUC and the pfu AUC data. The statistically significant negative correlations are indicated in red $\left({ }^{*} p<0.05 ;{ }^{* *} p<0.01\right)$ and the $r$ coefficient is indicated

vaccine decreased by $4 \log 10$ the peak of viral RNA levels in serum and was less efficient than purified $\mathrm{eGn}$ in oil-in-water adjuvant or than a viral replicon encoding for $\mathrm{Gn} / \mathrm{Gc}^{36}$ In the second study, clone 13 and the viral replicon induced full protection without detectable viral RNA in serum. ${ }^{34}$ However, it should be pointed out that the mean RNA copies per $\mathrm{ml}$ serum at the peak of infection of control sheep was close to $8 \log 10$ copies in the first study ${ }^{36}$ and to $10 \log 10$ copies in the second one, ${ }^{34}$ whereas it was above 12 $\log 10$ copies in our study, suggesting that our challenge conditions were more severe. We can speculate that a milder challenge would have improved the reduction of viral RNA load induced by our DNA vaccine (about 3 log10 here). Nevertheless, caution should be taken to compare these studies which were performed with different sheep breeds and different viral strains. Therefore, efforts should be made to better standardize across labs challenge experiments that should include a commercial vaccine as a reference to identify promising vaccines.

Higher levels of anti-eGn lgG were reached with peGn than with pscDEC-eGn and pscCD11c-eGn and correlated to protection. The higher $\mathrm{Ab}$ quantity could result from a higher expression of peGn in vivo, a hypothesis which is supported by the tendency for higher mRNA expression from peGn in skin; however, an analysis of protein expression from a large number of transfected skin biopsies would be necessary to further validate this hypothesis. In addition, untargeted eGn may be available to $B$ cells for a longer time than the DC-targeted eGn fusions which are likely to be rapidly secreted and readily internalized by the targeted receptor, a feature which has been proposed to reduce $A b$ responses while promoting T-cell responses. ${ }^{15}$

Besides quantity, the quality of the antibodies generated by our different DNA vaccines may also play a role in protection. In that respect, the fact that the anti-eGn antibodies induced by all three DNA vaccines were not neutralizing in vitro is puzzling. In addition, our DNA vaccines did not appear to prime for neutralizing antibody production since the DNA vaccinated sheep and control sheep showed similar levels at $10 \mathrm{dpc}$. In contrast, soluble eGn produced by drosophila cells and delivered in a oil-in-water adjuvant could induce neutralizing antibodies in sheep ${ }^{36}$ but in line with our finding, a DNA vaccine encoding untargeted eGn administered to mice using gene gun in skin conferred significant protection with weak induction of neutralizing antibodies. ${ }^{6}$ The eGn protein, related to its mode of expression by skin cells, may not be in an optimal conformation to induce neutralizing antibodies. Alternatively, the capacity of antibodies to neutralize the infectivity of RVFV may be different in the gold standard assay using VeroE6 cells and in the in vivo conditions, due to the likely difference of viral receptor engagement, as proposed in the case of flaviviruses. ${ }^{41}$ The anti-eGn antibodies induced by DNA vaccines may display anti-RVFV properties such as complement-dependent inhibition or Ab-dependent cellular cytotoxicity (ADCC) or 
phagocytosis. The ADCC efficacy depends on the Ab isotype and on the nature of the recognized epitope on the viral protein, as shown in the case of influenza. ${ }^{42}$ Untargeted eGn and eGn fused with scFv may differ in their respective capacities to generate such anti-viral antibodies. Assessment of the anti-RVFV Ab functions will require the development of suitable in vitro tests which would help to potentially identify better correlates of protection than anti-eGn Ab levels.

pscDEC-eGn did promote the IFNy T-cell responses in sheep, although it tended to be less expressed than peGn in sheep skin. In the context of DNA vaccination, DEC205 targeting appeared to have a positive effect on T-cell responses in several instances such as in mice ${ }^{12,13}$ and even cattle, ${ }^{43}$ but in other cases DEC205 targeting rather decreased the IFNY-producing T-cell response with regulatory T-cell induction. ${ }^{44,45}$ Here, the targeting of eGn to CD11c did not promote $T$-cell responses, although pscCD11c-eGn was at least as expressed as pscDEC-eGn. This is in contrast with results in mice where CD11c targeting strongly enhanced the IFNY T-cell responses. ${ }^{14}$ Therefore, the outcome of DEC205 and CD11C targeting of antigens expressed by DNA plasmids is inconsistent and difficult to predict for T-cell as well as for B-cell responses, ${ }^{12,44}$ in a given species and throughout species, and could depend on several parameters. Firstly, DC activation by the DNA delivery method is a prerequisite to induce strong T-cell responses, as the targeting of steady-state $\mathrm{DC}$ induce regulatory $\mathrm{T}$ cells and tolerance; ${ }^{46}$ therefore, the DNA delivery method, the combination with adjuvants and the duration of plasmid expression could all affect the efficacy of DC targeting. In the present work, we combined electroporation which has been proposed to have a strong adjuvant effect through inflammatory cytokine release and APC mobilization in skin, ${ }^{29}$ with a plasmid encoding ovine GMCSF, a largely used cytokine adjuvant in DNA vaccination including in sheep. ${ }^{8,28}$ Secondly, the nature of the targeted vaccine antigen, by its content in self-mimicking epitopes expanding regulatory Tcell responses, may affect effector T-cell priming. ${ }^{47}$ Thirdly, the same targeted orthologous receptor may have different subcellular routing pathways with variable kinetics in different species, impacting on the efficacy of major histocompatibility complex (MHC) class I or class II antigen presentation, ${ }^{17,48}$ and even in the same species, Ab targeted to different epitopes on the same molecule were shown to induce different responses. ${ }^{49}$ Finally, the distribution of expression of the targeted receptor among cell types and mononuclear phagocyte subsets in different species may affect the immune response, given the functional specialization of the different subsets. Indeed, in humans and ruminants, DEC205 expression on leukocyte populations is much broader than in mice and is found on B, T and NK lymphocytes. $^{50,51}$ In mice, CD11c is expressed primarily on DCs and also on some macrophages and natural killer (NK) cells, whereas in sheep CD11C is widely expressed on macrophages and B cells. ${ }^{52}$

To the best of our knowledge, our study is the first to demonstrate that a plasmid encoding for $\mathrm{eGn}$ induces a significant protection in sheep, indicating that an effective vaccine against RVFV could be produced based on DNA. However, our strategy needs to be further optimized. Indeed, for application in the field and especially in Africa, the minimum number of administrations and the plasmid dose to achieve protection should be established with downscaling experiments, and the mode of delivery should be adapted to mass vaccination, such as with a high pressure needle-free delivery system which has been shown to be as efficient as electroporation in pigs ${ }^{16}$ or possibly with new portable electroporation-based systems. ${ }^{53}$ The level of protection that we achieved is incomplete and complete immunity is desirable to protect the highly sensitive fetuses; however, a strong decrease in viral load would also decrease the risk of transmission to mosquito vectors. The immunity triggered by peGn might be further improved by adding other RVFV antigens such as by adding Gc (or $\mathrm{eGc}$ ) and/or $\mathrm{N}$ proteins. While the $\mathrm{T}$-cell response against $\mathrm{eGn}$ did not seem to impact protection, T-cell responses against other RVFV antigens may be of value. Better vectors driving higher antigen expression would be also desirable: indeed, differences in expression between vectors, as suggested by Fig. 2e, may have masked a positive effect of the targeting. Finally, as our results support the importance of anti-eGn IgG in protection, strategies to further improve this type of response should be privileged. In that respect, eGn could be targeted to receptors such as CLEC9A which is a particularly suitable targets for $A b$ response induction. ${ }^{54,55}$ CLEC9A is selectively expressed on CDC1s in different species including in sheep, ${ }^{23}$ and its targeting results in the prolonged antigen presentation favoring follicular helper T-cell development, strong germinal center formation, leading to high $\mathrm{Ab}$ responses of strong affinity. ${ }^{54}$ However, despite the existence of a CLEC9A ortholog in sheep and high expression of mRNA by $\mathrm{CDC} \mathrm{s}^{23}$ we were unable to generate any anti-CLEC9A mAb reacting with sheep cDC1s. Alternatively, XCR1 targeting, another receptor exclusively expressed by $\mathrm{CDC} 1 \mathrm{~s}$ across species, could also be used to successfully induce high anti-eGn lgG responses in sheep, as recently showed by us in pigs for promoting anti-influenza $\mathrm{M} 2 \mathrm{e}$ lgG responses. ${ }^{56}$

In conclusion, we showed that DNA vaccination is a very promising control strategy against RVFV in sheep. In addition, by using CD11C and DEC205 targeting which was detrimental to protection, we found that $A b$ responses against eGn, and not IFNY T-cell responses, are significant correlates of protection in sheep. This outcome of DC targeting related to its capacity to modulate immune responses could be extended to other pathogens for identifying immune correlates of protection in application species where mechanisms are difficult to investigate. In the case of RVFV, this DC-targeting strategy guides us towards the development of future vaccines optimized for induction of high levels of anti-eGn lgG whose anti-viral functions remain to be deciphered.

\section{MATERIALS AND METHODS}

\section{Abs and hydridomas}

The hybridomas producing the anti-ovine CD11c (mouse IgG1, OM1) and the anti-ruminant DEC205 (mouse IgG2b, CC98) mAbs were developed by INRA and the Pirbright Institute (former Institute for Animal Health (Compton, UK)) respectively. The mAbs used in this study are the antiCD1b (mouse IgG2a, Th97, WSU (USA)), the anti-CADM1 (chicken IgY, 3E1, CliniScience, \#CM004-3), the anti-bovine IFNy (CC330, Bio-Rad, \#MCA2112) and the biotinylated (biot)-anti-bovine IFNy (CC302, Bio-Rad, catalog \#MCA1783). The conjugated antibodies are the Alexa 647-conjugated (A647)-donkey anti-chicken IgY (Jackson ImmunoResearch, \#703-606-155) and the A488-donkey anti-mouse IgG (DAM) (Jackson ImmunoResearch, \#715-546-150), the A488-goat anti-mouse (GAM) IgG2a (Fisher Scientific, \#10114282), the phycoerythrin (PE)-GAM IgG1 (Fisher Scientific, \#10246642) and PE-GAM IgG2b (Fisher Scientific, \#10012802), and the horseradish peroxidase (HRP)-rabbit anti-mouse IgG (Sigma-Aldrich, \#A9044) and HRP-mouse anti-sheep lgG (Sigma-Aldrich, \#A9452). The anti-RVFV HMAF production was approved by the French Ministère de la Recherche et de l'Enseignement Supérieur under the number 02518.2 and was conducted at ANSES, Lyon, France. Balb/c mice were inoculated intraperitoneally with inactivated RVFV-infected mouse brain suspension in Freund's complete adjuvant on days 0 and 1, in Freund's incomplete adjuvant on days 7 and 14, and with a virulent suspension in Freund's incomplete adjuvant on days 21 and 28 . On day 25 , the mice were inoculated intraperitoneally with TG180 sarcoma cells. After 1 week, ascitic fluid was harvested.

\section{Cell lines}

Human embryonic kidney cells (HEK293, ATCC ${ }^{\circledR}$ CRL-1573) and African green monkey kidney cells (VeroE6, provided from Pierre Russo, Anses, Sophia-Antipolis Laboratory, Ruminant Pathology Unit, 06902, France) were maintained in culture with Dulbecco's modified Eagle's medium (DMEM, Life Technologies), supplemented with 5\% decomplemented fetal bovine serum (FBS) and antibiotic solution at $37^{\circ} \mathrm{C}$. Cell lines were checked 
to be free of mycoplasma contamination using a mycoplasma detection Kit (MycoAlert ${ }^{\mathrm{T} M}$, Lonza, Switzerland).

\section{RVFV strains}

The virulent strain RVFV 56/74, provided by Alejandro Brun (CISA-INIA), originally isolated from an infected $\mathrm{cow}^{31}$ was amplified on $\mathrm{C} 6 / 36$ insect cells and titrated on VeroE6 cells according to the Reed-Muench method. The attenuated RVFV virus MP-12 strain, ${ }^{57}$ provided by Michèle Bouloy (Paris Pasteur Institute), was amplified and titrated on VeroE6 cells.

\section{Construction and production of plasmids}

Genes encoding for the variable region of the murine heavy $(\mathrm{VH})$ and light (VL) chains of the anti-ruminant DEC205 and the anti-ovine CD11c mAb were amplified by PCR and subcloned in the PSK vector for sequencing (Synogene). The sequences were deposited on Bankit (https://www.ncbi. nlm.nih.gov) under the ID 2070859 (release on 1 September 2018). For each $\mathrm{mAb}$, the original $\mathrm{VH}$ signal peptide was fused to the $\mathrm{VH}$ and the $\mathrm{VL}$ fragments joined by a sequence encoding for a flexible linker $\left(\mathrm{G}_{4} \mathrm{~S}\right)_{4}$ to generate the scFv sequences. A sequence encoding for the ectodomain of the $\mathrm{Gn}$ glycoprotein (eGn) of the ZH501 strain was codon-optimized for expression in eukaryotic cells (Genecust, Luxembourg) and was fused in frame to the carboxy terminus of the scFv sequence with a $\left(\mathrm{G}_{4} \mathrm{~S}\right)_{3}$ linker. The eGn sequence spans from nucleotide (nt) 477 to $n t 1763$ (DQ380202.1) of the RVFV $M$ segment, and is devoid of transmembrane domains. The fusion constructs encompassing the VH signal peptide, scFv and eGn were cloned into a pcDNA3.1 expression vector (InvitroGen) and the resulting plasmids are designated as pscDEC-eGn and pscCD11c-eGn. In addition, fusion constructs with $m$ Cherry replacing eGn sequences were built as well as a construct including a control scFv, provided by Synogene (pscDECmCherry, pscCD11c-mCherry and pscCtrl-mCherry). A plasmid encoding for untargeted eGn was generated (peGn). peGn includes the nt 411 to nt 1763 codon-optimized sequence of the $M$ segment with the natural signal peptide of $\mathrm{Gn}^{58}$ cloned into a pcDNA4-V5His expression vector (InvitroGen) (Genecust). The plasmid encoding for ovine GM-CSF (pGM-CSF) has been previously described.$^{28}$ A firefly luciferase expression plasmid (pLuc) was kindly provided by Stéphane Biacchesi (INRA, France). The sequence encoding the RVFV $\mathrm{N}$ nucleoprotein was cloned into the expression vector pMT/BiP/SNAP-His. Plasmid productions for immunization were prepared using Endofree ${ }^{\circledast}$ Plasmid Giga Kits (Macherey-Nagel) and were stored at $-20^{\circ} \mathrm{C}$ until use.

\section{Plasmid transfection in vitro}

HEK293 cells $\left(5 \times 10^{6}\right.$ cells) were transiently transfected with JetPRIME ${ }^{\circledast}$ (Polyplus, USA) according to the manufacturer's protocol. Briefly, $10 \mu \mathrm{g}$ of peGn, pscDEC-eGn, pscCD11c-eGn or pcDNA3.1 (negative control) were mixed with $20 \mu \mathrm{l}$ of polymer reagent and added to the cells. The supernatants and cells were harvested at $72 \mathrm{~h}$ post transfection.

\section{Cell lysate and supernatant preparation}

Cell pellets of transfected HEK293 cells or MP-12-infected VeroE6 cells were lysed in borate lysis buffer ( $1 \%$ Triton X-100, $50 \mathrm{mM}$ borate, $150 \mathrm{mM} \mathrm{NaCl}$, $\mathrm{pH}$ 9)+protease inhibitors at a 1:9 volume ratio (pellet/buffer), the lysates were sonicated, aliquoted and kept frozen. The transfected cell supernatants were concentrated 20 to 40 times with Amicon ${ }^{\circledR}$ Ultra centrifugal filter $30 \mathrm{~K}$ (Millipore) and a protease inhibitor cocktail was added.

\section{Western blots and dot blots}

Cell lysates were analyzed by western blot following a $12 \%$ sodium dodecyl sulfate-polyacrylamide gel electrophoresis (SDS-PAGE) under reducing conditions and cell supernatants were analyzed by dot blot under nonreducing conditions. Blots were incubated overnight at $4{ }^{\circ} \mathrm{C}$ with anti-RVFV HMAF in a 1:1000 dilution in phosphate-buffered saline (PBS) $+10 \%$ fat-free milk (w/v) followed by HRP-rabbit anti-mouse lgG Ab. Immunoreactive bands were visualized with $\mathrm{ECL}$ substrate (SuperSignal ${ }^{\mathrm{TM}}$ West Femto, Thermofisher) and dots were visualized with 3,3'-diaminobenzidine substrate (Sigma-Aldrich). Image acquisitions were done with Chemidoc imaging system (Bio-Rad). The blot experiment was performed with respect to the ethical guidelines and the technical protocols conducted at ANSES laboratory in Lyon, France.
Lymph duct cannulation and low-density lymph cell collection The prescapular lymph duct of Prealpes sheep was cannulated to collect pseudo-afferent lymph cells draining from the skin, as previously described. ${ }^{59}$ The cannulated sheep were housed at the Unite Commune d'Expérimentation Animale in Jouy-en-Josas, France. This protocol was done under compliance with national legislation on animal experimentation and was approved by the Committee on the Ethics of Animal Experiments of the INRA research center in Jouy-en-Josas (COMETHEA) under the number 11-019.

\section{Assessment of plasmid transfection efficacy in sheep skin}

Plasmid administration in sheep skin was performed on Prealpes sheep (2 to 12 months old) under general anesthesia $(2 \mathrm{mg} / \mathrm{kg}$ xylazine and $10 \mathrm{mg} /$ $\mathrm{kg}$ ketamine, intramuscular route). Immediately after intradermal injection of DNA in the left and right inner front leg zones, SEP was performed on the site of injection using the CUY 21 EDIT system (BEX, Tokyo, Japan). Disk electrodes $(10 \mathrm{~mm})$ were loaded with conductive gel (Alcyon, France) and 6 electric pulses were applied during $10 \mathrm{~ms}$ with $90 \mathrm{~ms}$ interval. In order to evaluate the $\mathrm{V} / \mathrm{cm}$ parameter leading to optimal transfection in sheep skin, a pLuc reporter vector $(100 \mu \mathrm{g})$ was injected intradermally and the injected skin areas were subjected to SEP with escalating pulses from 0 to $1331 \mathrm{~V} /$ $\mathrm{cm}$. After 2 days, skin punch biopsies were harvested and lysed with $150 \mu \mathrm{l}$ of lysis reagent (Luciferase Assay System E1500, Promega). Bioluminescence was measured using the In Vivo Imaging System (IVIS-200, Xenogen, UK) after adding $100 \mu \mathrm{l}$ luciferine substrate. The optimal V/cm was found to be $532 \mathrm{~V} / \mathrm{cm}$ and was used in all subsequent experiments (supplementary material 1). This experiment was approved by the COMETHEA ethic committee under the number 201504211534406 (APAFIS\#520) in accordance with national guidelines on animal use and performed at the Unité Commune d'Expérimentation Animale UCEA-INRA in Bressonvilliers, France. In order to compare the expression of the different vaccine plasmids in sheep skin in vivo, plasmids $(100 \mu \mathrm{g})$ were injected intradermally and subjected to SEP $(532 \mathrm{~V} / \mathrm{cm})$. After 2 days, sheep were killed, 4 skin biopsies were collected from each injection site and frozen in liquid nitrogen. This experiment was approved by the French ethical committee CEEA Val de Loire, received the authorization number 04150.02 in accordance with national guidelines on animal use and was conducted at the Plate-Forme d'Infectiologie Expérimentale PFIE-INRA, Nouzilly, France.

\section{Immunizations and RVFV challenge}

The experiment was approved by the Spanish ethical committee Department Medi Ambient i Habitatge de la Generalitat de Catalunya CE-8820 and was conducted at IRTA-CReSA, Barcelona, Spain, under compliance to Spanish biosafety and ethic rules. Lambs (2 months old) were equally distributed in both sexes and were housed in the IRTA-CReSA experimental farm in Lerida, Spain, under A-BSL1. Lambs were randomly divided into 4 groups of 8 lambs and the vaccination was performed 3 times at 4 week intervals $(0,4,8)$ under general anesthesia $(2 \mathrm{mg} / \mathrm{kg}$ zoletil, intramuscular route); 8 animals per group is classically used in vaccine studies. Then, $400 \mu \mathrm{g}$ of peGn, pscDEC-eGn or pscCD11c-eGn were injected intradermally together with $400 \mu \mathrm{g}$ of plasmid adjuvant (pGM-CSF), followed by SEP as described above. A non-vaccinated control group (PBS plus pGM-CSF) was included. Sera were collected at weeks $0,4,8$ and 12 post immunization and PBMCs were harvested at week 12 to analyze the T-cell response. The immunized sheep were moved to a A-BSL3 to perform the challenge at day 91 after the first immunization with $10^{6}$ $\mathrm{TCID}_{50}$ of the RVFV 56/74 strain in $2 \mathrm{ml}$ volume by subcutaneous route. After challenge, the clinical signs were daily monitored in a blinded manner. The daily clinical score per sheep was established by monitoring anorexia, eye aspect, gait, lack of rumination, mucosa aspect, prostration, respiratory movement, tremor ( 1 to 3 depending of severity) and death (15) (supplementary material 2). An individual clinical score per sheep was obtained by adding the daily values over 20 days. The final global score per group was the addition of each individual score. Serum was collected at 0 , $1,2,3,4,5,6,8,10,12,14,16,18,20 \mathrm{dpc}$. At the end of the study, the animals were killed by an overdose of sodium pentothal.

\section{Low-density lymph cells and PBMC isolation}

Low-density lymph cells were enriched over an optiprep gradient as previously described by our group, ${ }^{59}$ frozen in FCS+10\% DMSO using a Nalgene Mr. Frosty Cryo $1{ }^{\circ} \mathrm{C}$ Freezing Container and kept in liquid nitrogen. PBMCs were isolated in the A-BSL3 IRTA-CReSA's facility using 
$8 \mathrm{ml}$ Vacutainer ${ }^{\circledast}$ CPT $^{\mathrm{TM}}$ (BD Bioscience) by centrifugation at $1800 \times g$ for $35 \mathrm{~min}$ at $25^{\circ} \mathrm{C}$. Tubes were shipped overnight at $4{ }^{\circ} \mathrm{C}$ to INRA, Jouy-enJosas, France. Upon arrival, PBMCs were washed with PBS+1.3 mM citrate and re-suspended in X-vivo medium (Ozyme, France) supplemented with $50 \%$ FBS for overnight recovery at $4{ }^{\circ} \mathrm{C}$.

\section{Staining of lymph dendritic cell and flow cytometry analysis}

Low-density lymph cells were thawed and incubated on ice with PBS supplemented with $4 \%$ horse serum for $30 \mathrm{~min}$. To analyze DEC205 and CD11c expression on DC subsets, lymph cells were labeled with a mix of primary $\mathrm{mAbs}(2 \mu \mathrm{g} / \mathrm{ml})$ for $30 \mathrm{~min}$, being either anti-CD1b+anti-CADM1 +anti-DEC205 mAbs, or anti-CD1b+anti-CADM1+anti-CD11c mAbs. Appropriate isotype control mAbs were used. Cells were washed and finally incubated with a mix of secondary Abs $(1: 200)$ being respectively A488-GAM lgG2a+A647-anti-chicken IgY+PE-GAM IgG2b or A488-GAM $\lg 2 \mathrm{a}+\mathrm{A} 647$-anti-chicken $\lg Y+P E-G A M \operatorname{lgG} 1$. To analyze the binding of scDEC-mCherry and scCD11c-mCherry, the fusion proteins $(50 \mu \mathrm{g} / \mathrm{ml})$ +anti-CD1b+anti-CADM1 mAbs were incubated with lymph cells on ice for $30 \mathrm{~min}$. A scCtrl-mCherry provided by Synogene was used as control. Cells were washed and incubated with PE-GAM IgG1+A488-GAM IgG2a. To analyze the binding of scDEC-eGn and scCD11c-eGn on low-density lymph cells, the concentrated supernatants of HEK293 transfected cells with peGn, pscDEC-eGn, pscCD11c-eGn and pcDNA3.1 (negative control) were incubated with cells for $2 \mathrm{~h}$ at $4{ }^{\circ} \mathrm{C}$. Cells were then incubated with antiRVFV HMAF (1:1000) and finally with A488-DAM IgG (1:200). Dead cells were excluded by 4',6-diamidino-2-phenylindole (DAPI) staining. Flow cytometry acquisitions were done with a LSR Fortessa flow cytometer (Becton Dickinson) and results were analyzed using FlowJo 10.0.6 software.

\section{Evaluation of eGn-specific T-cell responses by ELISPOT}

IFNY-secreting $T$ cells were detected using polyvinylidene difluoride membrane-bottomed 96 -well plates (Multiscreen ${ }^{\circledast}{ }_{\mathrm{HTS}}$, Millipore) coated with $15 \mu \mathrm{g} / \mathrm{ml}$ anti-bovine IFNY (capture $\mathrm{mAb}$ ) in PBS. PBMCs $\left(2.5 \times 10^{5}\right)$ were added to wells and re-stimulated with $5 \mu \mathrm{g} / \mathrm{ml}$ overlapping peptides (20 mers, offset 8, Mimotopes, Australia) covering the N-terminal (AEDPHL [...] LLPDSF) and C-terminal (VCFEHK [...] NYQCHT) amino-acid sequence of the eGn protein in X-vivo medium (Ozyme, France) supplemented with $2 \% \mathrm{FBS}$ and antibiotic solution at $37^{\circ} \mathrm{C}$ for $18 \mathrm{~h}$. The overlapping peptides were divided in 2 pools initially diluted in $\mathrm{H}_{2} \mathrm{O} /$ acetonitril $(5 \mathrm{mg} / \mathrm{ml})$ and distributed in $5 \mu \mathrm{l}$ per well to have $1 \mu \mathrm{g}$ of each peptide per well and avoid any toxicity of the $\mathrm{H}_{2} \mathrm{O}$ /acetonitril diluent. In addition, cells incubated with $5 \mu \mathrm{l}$ of $\mathrm{H}_{2} \mathrm{O}$ /acetonitril peptide diluent (1:1 volume) and with an irrelevant peptide derived from the HIV polymerase (IKDFHVYFRESRDALWKGPG) were used as negative controls. Cells cultured with ConA $(25 \mu \mathrm{g} / \mathrm{ml})$ were used as positive controls. Each condition was tested in duplicated wells. IFNY-secreting T cells were revealed using biot-anti-bovine IFNY mAb at $0.5 \mu \mathrm{g} / \mathrm{ml}$ followed by alkaline phosphatase-conjugated streptavidin (Mabtech, Sweden) and BCIP/NBT reagent (Life Technologies). Plates were automatically counted using the iSPOT AID ELISPOT reader. IFNY spot counts were considered eGn specific when both duplicated well counts from cells stimulated with eGn peptides were strictly superior to the duplicated well counts from cells stimulated with irrelevant peptide. The net mean of the IFNy spot counts were obtained by subtracting the mean counts from wells stimulated with irrelevant peptides out of the mean counts from wells stimulated with eGn peptides.

Evaluation of anti-eGn, anti-N and anti-RVFV responses by ELISA High-binding 96-well plates Nunc Maxisorp ${ }^{\mathrm{TM}}$ (ThermoFisher) were coated overnight at $4{ }^{\circ} \mathrm{C}$ either with $100 \mu$ lysates from MP-12-infected VeroE6 cells or from HEK293 cells transfected with peGn or with SNAP-tag purified $\mathrm{N}$ protein, which was produced using a Drosophila expression system and kindly provided by Philippe Despres (Paris Pasteur Institute, France). The same lysates and protein batch was used in all experiments. The same positive and negative sheep sera were used to validate each plate. Sera were diluted 1:100 in PBS $+2 \%$ fat-free milk $(\mathrm{w} / \mathrm{v})$ and incubated on the coated plates at $37^{\circ} \mathrm{C}$ for $1 \mathrm{~h}$. The plates were subsequently incubated with HRP-mouse anti-sheep IgG in a $1: 4000$ dilution at $37^{\circ} \mathrm{C}$ for $1 \mathrm{~h}$. HRP enzymatic activity was revealed using TMB $\left(3,3^{\prime}, 5,5^{\prime}\right.$-tetramethylbenzidine) substrate. Optical density was measured at $450 \mathrm{~nm}$ using a TECAN microplate reader. Corrected OD values were obtained by subtracting the signal of wells coated with lysates from uninfected VeroE6 cells, from HEK293 cell transfected with pcDNA3.1, or coated with SNAP-tag. To calculate anti-eGn IgG end-point titers, 1:100 diluted sera were serially diluted 1:1 and the ELISA was processed as described above. End-point titers were calculated with a regression analysis plotting dilution versus $A_{450}$ as the highest dilution giving twice the absorbance of the mean of negative control wells.

\section{RNA extraction from serum and transfected skin samples}

Total RNA was extracted from post-infection sera with QIAMP kit (Qiagen) according to the manufacturer's instructions and eluted in $60 \mu \mathrm{l}$ RNase-free water using QIACube (Qiagen). Total RNA was extracted from skin biopsies collected from the inoculation sites (about $250 \mathrm{mg}$ per site) using $10 \mathrm{ml}$ TRIzol LS reagent (Thermo Fisher Scientific) and homogenization with Ultra-Turrax T8. The first RNA extract was purified using a RNeasy MinElute cleanup column. The $1 \mu \mathrm{g}$ purified RNA was treated twice with $5 \mu \mathrm{l}$ of RNAse-free DNAse (30 Kunitz units, Qiagen) in order to remove any residual DNA plasmid from the injection and subjected to a final purification on column.

\section{Gn RNA quantification by qRT-PCR}

For quantification of viral RNA copies per $\mathrm{ml}$ sera, a one-step qRT-PCR was performed using the SuperScript III Platinum One-Step qRT-PCR kit (Invitrogen) as previously described ${ }^{60}$ Primers were designed to amplify a nt 1164 to nt $1258 \mathrm{Gn}$ sequence of the $M$ segment: forward primer $5^{\prime}$ AAA GGA ACA ATG GAC TCT GGT CA-3', reverse primer 5'- CAC TTC TTA CTA CCA TGT CCT CCA AT-3'. A fluorescent probe was designed that hybridizes to the specific PCR product: FAM 5'- AAA GCT TTG ATA TCT CTC AGT GCC CCA A-3' TAMRA. A standard corresponding to nt 1135 to nt 1286 of the $M$ segment was synthetized using the Riboprobe ${ }^{\circledR}$ In Vitro Transcription Systems (Promega). The qRT-PCR was done on $3 \mu \mathrm{l}$ of the serum elution and the program was $45^{\circ} \mathrm{C}$ for $30 \mathrm{~min}, 95^{\circ} \mathrm{C}$ for $5 \mathrm{~min}$, linked to 45 amplification cycles of $95^{\circ} \mathrm{C}$ for $5 \mathrm{~s}$ and $57^{\circ} \mathrm{C}$ for $35 \mathrm{~s}$, and cooling at $37^{\circ} \mathrm{C}$ for $30 \mathrm{~s}$. TaqMan run of experimental samples contained at least two replicates, a known positive control (RVFV ZH501 strain), a negative control (an other Phlebovirus RNA) and nuclease-free water. The number of viral RNA copies/ml in each serum sample was determined using the Gn RNA standard calibration curve. For each animal, the AUC of the viral RNA copies per $\mathrm{ml}$ over 10 days (viral RNA AUC) was calculated. For the detection of Gn mRNA in transfected skin biopsies, a two-step qRT-PCR was performed. The reverse transcription was done using the TaqMan ${ }^{\circledR}$ Reverse Transcription Reagents (Applied Biosystems). In order to check that the transfected skin RNA did not include detectable residual plasmids, reverse-transcribed ( $3 \mathrm{ng}$ estimated $\mathrm{CDNA}$ ) and non-reverse transcribed RNA (3 ng RNA) were subjected to PCR reaction with the iTaq UniverSYBR Green (Bio-Rad) for amplifying a $\mathrm{Gn}$ fragment with forward primer $5^{\prime}$ - AGT GCG ATG GGC AGT TGT C-3' (M segment, codon-optimized sequence) and reverse primer $5^{\prime}$ - TTC TTG AAC ACG GCA AAT GG-3'. The PCR cycling conditions were $95^{\circ} \mathrm{C}$ for $10 \mathrm{~min}$, linked to 40 cycles of $95^{\circ} \mathrm{C}$ for $15 \mathrm{~s}$ and $60^{\circ} \mathrm{C}$ for $1 \mathrm{~min}$. No residual plasmid could be detected in any of the RNA samples. The relative expression of Gn mRNA in transfected skin samples was normalized using the qRT-PCR signals obtained with the ovine ribosomal protein 24 (RSP24) gene which is a stably expressed reference gene in sheep cells. ${ }^{61}$

\section{Virus titration by plaque assay}

The sheep sera were serially diluted in DMEM medium and inoculated onto VeroE6 cells in 12-well plates for a plaque assay over 5 days. The pfu were revealed by $1 \%(\mathrm{w} / \mathrm{v})$ violet crystal and counted. For each animal, the integrated pfu load corresponding to the AUC of the number of pfu per $\mathrm{ml}$ serum over 8 days was calculated (pfu AUC).

\section{Plaque reduction assay}

Neutralizing antibody was measured using plaque reduction and neutralization test. Sera were diluted 1:10 to $1: 10^{4}$ in DMEM and incubated with 100 pfu of MP- 12 virus at $37^{\circ} \mathrm{C}$ for $1 \mathrm{~h}$ to enable neutralization to occur. Next, the virus and the serum mix $(500 \mu l)$ were added to VeroE6 cell monolayers for a plaque assay. The neutralizing antibody titers were established as the last dilution which inhibited $50 \%$ of the foci number per well compared to virus-only control titration.

\section{Statistical and correlation analysis}

Data were analyzed with the GraphPad Prism 6.0 software. To compare the T-cell responses, the anti-eGn IgG titers, the NAb titers at day 10 , the viral 
RNA at day 3, the pfu at day 2, the viral RNA AUC, the pfu AUC values and the luciferase signals between the two groups, we used the unpaired nonparametric Mann-Whitney test, as the data did not pass the normality test (Kolmogorov-Smirnov test). The paired parametric $t$-test was used to compare the antibody responses at different time points versus at day 0 within the same group. A two-way analysis of variance (ANOVA) test followed by Bonferroni's correction was used to compare the OD values of the $A b$ responses and the body temperature between immunized groups each day after infection, as the data distribution passed normality test. In most cases the standard deviations of the data were statistically similar between vaccinated groups (Brown-Forsythe test) but they were different between the vaccinated and the non-vaccinated groups. The correlation analysis between the immune response data versus the individual clinical scores, the viral RNA AUC and the pfu AUC was done with the bilateral Spearman's non-parametric test. The PCA was done with 31 factors and included the individual viral RNA copies/ml at 2, 3, 4, 5 and $6 \mathrm{dpc}$, the viral RNA AUC, the individual $\mathrm{pfu} / \mathrm{ml}$ at 2,3 and $4 \mathrm{dpc}$, the pfu AUC, the individual clinical scores, the integrated temperature measurements, the Tcell responses at week 12, the anti-eGn IgG OD signals at 0, 5, 6, 8, 10 and $12 \mathrm{dpc}$, the anti-N IgG OD signals at 5, 6, 8, 10 and $12 \mathrm{dpc}$, the anti-RVFV IgG OD signals at $0,5,6,8,10$ and $12 \mathrm{dpc}$ and the NAb titers at $10 \mathrm{dpc}$. PCA graphics has been produced using FactoMineR R package (http:// factominer.free.fr/).

\section{Data availability}

All datasets and tools developed by the authors are available to readers. Samples for RVFV-infected sheep and vaccinated sheep will require legal authorization from the Agence Nationale de la Sécurite du Médicament (ANSM), as RVFV is a "select agent" in this country. The ScFv sequences were deposited on Bankit (https://www.ncbi.nlm.nih.gov) under the ID 2070859 (release on 1 September 2018).

\section{ACKNOWLEDGEMENTS}

We are grateful to Dany Leguere, Guillaume Martin, Olivier Boulesteix and Sylvain Breton for their efficient participation in the experiments conducted at the PFIE and to Pierre Sarradin for managing the regulatory aspects. We are grateful to Noémie Perrot for the experiment conducted at UCEA. We thank the personnel of the IRTA Experimental farm, i.e., Jordi Charles (IRTA-CReSA) as the coordinator in charge, Rafel Martos Sánchez (Freevet Bienestar Animal, C/ Jose Marco 15 Bajo B, 22500 Binefar, Spain) as the veterinary surgeon in charge and Miquel Molins (Laboratorio de Sanidad Animal de Catalunya (LSA, Avinguda de l'Alcalde Rovira Roure, 191, 25198 Lleida, Spain), the laboratory director, who kindly ceded its facilities to process recently taken samples from the experimental farm. We thank the personnel of the IRTA-CReSA facilities, i.e., Raquel Rivas who was in charge of the RVFV stock preparation, Nuria Navarro who processed sheep samples at under strict biosafety measures, the personnel of bio-containment unit and David Solanes as the Biosafety Officer and Head Coordinator of Technical Services. We are grateful to Alejandro Brun (CISA-INIA, Centro de Investigación en Sanidad Animal, 28130 Madrid, Spain) who kindly agreed to use the RVFV strain at IRTA-CReSA for this study. We thank Michèle Bouloy (Paris Pasteur Institute, France) for the MP-12 strain, Stéphane Biacchesi (VIMINRA-Université Paris-Saclay, France) for the pLuc plasmid and Philippe Desprès (Paris Pasteur Institute, France) for the purified N protein. We thank the Synogene team for their excellent technical support and follow-up with the scFv constructs. The work was supported by the grant ANR ASTRID RIFTVAC ANR-12-ASTR-0006-01, financed by the Direction Générale de I'Armement (France). T.C. was supported by a joint INRAANSES fellowship.

\section{AUTHOR CONTRIBUTIONS}

T.C.: performed most of the experiments, organized experiments, analyzed all results, prepared all the figures and tables and wrote parts of the manuscript. I.S.-C.: designed and directed the study, coordinated the financial support and the experiments, wrote the request to the ethic committee, performed experiments, analyzed results, wrote parts and supervised the writing of the manuscript. P.M.: designed and directed the study and analyzed results. A.F.-R. and C.N.P.: designed and contributed to experiments. S.L. and C.U.: performed experiments (ELISA and qRT-PCR). L.J.: performed statistical analyses. C.B., E.B., E.V., J.P. and N.B.: performed animal experiments. V.C. performed analyses (ELISPOTS).

\section{ADDITIONAL INFORMATION}

Supplementary information accompanies the paper on the npj Vaccines website (https://doi.org/10.1038/s41541-018-0052-x).

Competing interests: The authors declare no competing interests.

Publisher's note: Springer Nature remains neutral with regard to jurisdictional claims in published maps and institutional affiliations.

\section{REFERENCES}

1. Pepin, M., Bouloy, M., Bird, B., Kemp, A. \& Paweska, J. Rift Valley fever virus (Bunyaviridae: Phlebovirus): an update on pathogenesis, molecular epidemiology, vectors, diagnostics and prevention. Vet. Res. 41, 61-66 (2010).

2. Schmaljohn, C. S. et al. Baculovirus expression of the $M$ genome segment of Rift Valley fever virus and examination of antigenic and immunogenic properties of the expressed proteins. Virology 170, 184-192 (1989).

3. Wu, Y. et al. Structures of phlebovirus glycoprotein $\mathrm{Gn}$ and identification of a neutralizing antibody epitope. Proc. Natl Acad. Sci. USA 114, E7564-E7573 (2017).

4. Besselaar, T. G. \& Blackburn, N. K. Topological mapping of antigenic sites on the Rift Valley fever virus envelope glycoproteins using monoclonal antibodies. Arch. Virol. 121, 111-124 (1991).

5. de Boer, S. M. et al. Rift Valley fever virus subunit vaccines confer complete protection against a lethal virus challenge. Vaccine 28, 2330-2339 (2010).

6. Bhardwaj, N., Heise, M. T. \& Ross, T. M. Vaccination with DNA plasmids expressing Gn coupled to C3d or alphavirus replicons expressing Gn protects mice against Rift Valley fever virus. PLoS. Negl. Trop. Dis. 4, e725 (2010).

7. Lopez-Gil, E. et al. A single immunization with MVA expressing GnGc glycoproteins promotes epitope-specific CD8+-T cell activation and protects immunecompetent mice against a lethal RVFV infection. PLoS Negl. Trop. Dis. 7, e2309 (2013).

8. Saade, F. \& Petrovsky, N. Technologies for enhanced efficacy of DNA vaccines. Expert. Rev. Vaccin. 11, 189-209 (2012).

9. Redding, L. \& Weiner, D. B. DNA vaccines in veterinary use. Expert. Rev. Vaccin. 8, 1251-1276 (2009).

10. Tregoning, J. S. \& Kinnear, E. Using Plasmids as DNA Vaccines for Infectious Diseases. Microbiol. Spectr. 2, https://doi.org/10.1128/microbiolspec.PLAS-00282014 (2014).

11. Gothelf, A. \& Gehl, J. What you always needed to know about electroporation based DNA vaccines. Human. Vaccin. Immunother. 8, 1694-1702 (2012).

12. Nchinda, G. et al. The efficacy of DNA vaccination is enhanced in mice by targeting the encoded protein to dendritic cells. J. Clin. Invest. 118, 1427-1436 (2008).

13. Cao, J. et al. DNA vaccines targeting the encoded antigens to dendritic cells induce potent antitumor immunity in mice. BMC Immunol. 14, 39 (2013).

14. Wang, Q., Cao, W., Yang, Z. G. \& Zhao, G. F. DC targeting DNA vaccines induce protective and therapeutic antitumor immunity in mice. Int. J. Clin. Exp. Med. 8, 17565-17577 (2015)

15. Gudjonsson, A. et al. Targeting influenza virus hemagglutinin to Xcr1+dendritic cells in the absence of receptor-mediated endocytosis enhances protective antibody responses. J. Immunol. 198, 2785-2795 (2017).

16. Grodeland, G. et al. Antigen targeting to human HLA class II molecules increases efficacy of DNA vaccination. J. Immunol. 197, 3575-3585 (2016).

17. Reuter, A. et al. Criteria for dendritic cell receptor selection for efficient antibodytargeted vaccination. J. Immunol. 194, 2696-2705 (2015).

18. Schlitzer, A., McGovern, N. \& Ginhoux, F. Dendritic cells and monocyte-derived cells: two complementary and integrated functional systems. Semin. Cell Dev. Biol. 41, 9-22 (2015).

19. Guilliams, M. et al. Unsupervised high-dimensional analysis aligns dendritic cells across tissues and species. Immunity 45, 669-684 (2016).

20. Vu Manh, T. P. et al. Defining mononuclear phagocyte subset homology across several distant warm-blooded vertebrates through comparative transcriptomics. Front. Immunol. 6, 299 (2015).

21. Macri, C., Dumont, C., Johnston, A. P. \& Mintern, J. D. Targeting dendritic cells: a promising strategy to improve vaccine effectiveness. Clin. Transl. Immunol. 5, e66 (2016).

22. Caminschi, I., Maraskovsky, E. \& Heath, W. R. Targeting dendritic cells in vivo for cancer therapy. Front. Immunol. 3, 13 (2012).

23. Contreras, V. et al. Existence of CD8\{alpha\}-like dendritic cells with a conserved functional specialization and a common molecular signature in distant mammalian species. J. Immunol. 185, 3313-3325 (2010).

24. Apostolico, J. S. et al. Dendritic cell targeting effectively boosts $T$ cell responses elicited by an HIV multiepitope DNA vaccine. Front. Immunol. 8, 101 (2017). 
25. Hua, Y. et al. Enhanced humoral and CD8+T cell immunity in mice vaccinated by DNA vaccine against human respiratory syncytial virus through targeting the encoded F protein to dendritic cells. Int. Immunopharmacol. 46, 62-69 (2017).

26. White, A. L. et al. Ligation of CD11c during vaccination promotes germinal centre induction and robust humoral responses without adjuvant. Immunology 131, 141-151 (2010).

27. Alvarez, B. et al. Antigen targeting to APC: from mice to veterinary species. Dev. Comp. Immunol. 41, 153-163 (2013).

28. Niborski, V. et al. Efficacy of particle-based DNA delivery for vaccination of sheep against FMDV. Vaccine 24, 7204-7213 (2006).

29. Todorova, B. et al. Electroporation as a vaccine delivery system and a natural adjuvant to intradermal administration of plasmid DNA in macaques. Sci. Rep. 7, 4122 (2017).

30. Flacher, V. et al. Skin langerin+dendritic cells transport intradermally injected anti-DEC-205 antibodies but are not essential for subsequent cytotoxic CD8+T cell responses. J. Immunol. 188, 2146-2155 (2012).

31. Busquets, N. et al. Experimental infection of young adult European breed sheep with Rift Valley fever virus field isolates. Vector Borne Zoonotic Dis. 10, 689-696 (2010).

32. Faburay, B., LaBeaud, A. D., McVey, D. S., Wilson, W. C. \& Richt, J. A. Current status of Rift Valley fever vaccine development. Vaccines (Basel) 5, pii: E29 (2017).

33. Makoschey, B. et al. Rift Valley fever vaccine virus clone 13 is able to cross the ovine placental barrier associated with foetal infections, malformations, and stillbirths. PLoS Negl. Trop. Dis. 10, e0004550 (2016).

34. Kortekaas, J. et al. Comparative efficacy of two next-generation Rift Valley fever vaccines. Vaccine 32, 4901-4908 (2014).

35. Ly, H. J. et al. Attenuation and protective efficacy of Rift Valley fever phlebovirus rMP12-GM50 strain. Vaccine 35, 6634-6642 (2017)

36. Kortekaas, J. et al. Efficacy of three candidate Rift Valley fever vaccines in sheep. Vaccine 30, 3423-3429 (2012).

37. Faburay, B. et al. A recombinant Rift Valley fever virus glycoprotein subunit vaccine confers full protection against Rift Valley fever challenge in sheep. Sci. Rep. 6, 27719 (2016).

38. Warimwe, G. M. et al. Chimpanzee adenovirus vaccine provides multispecies protection against Rift Valley fever. Sci. Rep. 6, 20617 (2016).

39. Busquets, N. et al. Efficacy assessment of an MVA vectored Rift Valley Fever vaccine in lambs. Antivir. Res. 108, 165-172 (2014).

40. Lorenzo, G., Martin-Folgar, R., Rodriguez, F. \& Brun, A. Priming with DNA plasmids encoding the nucleocapsid protein and glycoprotein precursors from Rift Valley fever virus accelerates the immune responses induced by an attenuated vaccine in sheep. Vaccine 26, 5255-5262 (2008).

41. Mukherjee, $\mathrm{S}$. et al. Mechanism and significance of cell type-dependent neutralization of flaviviruses. J. Virol. 88, 7210-7220 (2014).

42. He, W. et al. Epitope specificity plays a critical role in regulating antibodydependent cell-mediated cytotoxicity against influenza A virus. Proc. Natl. Acad. Sci. USA 113, 11931-11936 (2016).

43. Njongmeta, L. M. et al. CD205 antigen targeting combined with dendritic cell recruitment factors and antigen-linked CD40L activation primes and expands significant antigen-specific antibody and CD4(+) T cell responses following DNA vaccination of outbred animals. Vaccine 30, 1624-1635 (2012).

44. Niezold, T. et al. DNA vaccines encoding DEC205-targeted antigens: immunity or tolerance? Immunology 145, 519-533 (2015).

45. Tenbusch, M. et al. Immunogenicity of DNA vaccines encoding simian immunodeficiency virus antigen targeted to dendritic cells in rhesus macaques. PLoS One 7, e39038 (2012)

46. Hawiger, D. et al. Dendritic cells induce peripheral T cell unresponsiveness under steady state conditions in vivo. J. Exp. Med. 194, 769-779 (2001).
47. Losikoff, P. T. et al. HCV epitope, homologous to multiple human protein sequences, induces a regulatory $T$ cell response in infected patients. J. Hepatol. 62, 48-55 (2015)

48. Macri, C. et al. Antibody-mediated targeting of antigen to C-type lectin-like receptors Clec9A and Clec12A elicits different vaccination outcomes. Mol. Immunol. 81, 143-150 (2016).

49. Dakappagari, N. et al. Internalizing antibodies to the C-type lectins, L-SIGN and DC-SIGN, inhibit viral glycoprotein binding and deliver antigen to human dendritic cells for the induction of T cell responses. J. Immunol. 176, 426-440 (2006).

50. Kato, M. et al. Expression of human DEC-205 (CD205) multilectin receptor on leukocytes. Int. Immunol. 18, 857-869 (2006).

51. Gliddon, D. R., Hope, J. C., Brooke, G. P. \& Howard, C. J. DEC-205 expression on migrating dendritic cells in afferent lymph. Immunology 111, 262-272 (2004).

52. Chevallier, N. et al. B-1-like cells exist in sheep. Characterization of their phenotype and behaviour. Immunology 95, 178-184 (1998).

53. McCoy, J. R. et al. A multi-head intradermal electroporation device allows for tailored and increased dose DNA vaccine delivery to the skin. Hum. Vaccin. Immunother. 11, 746-754 (2015).

54. Lahoud, M. H. et al. Targeting antigen to mouse dendritic cells via Clec9A induces potent CD4 T cell responses biased toward a follicular helper phenotype. J. Immunol. 187, 842-850 (2011).

55. Park, H. Y. et al. Evolution of B cell responses to Clec9A-targeted antigen. J. Immunol. 191, 4919-4925 (2013).

56. Deloizy, C. et al. The anti-influenza M2e antibody response is promoted by XCR1 targeting in pig skin. Sci. Rep. 7, 7639 (2017).

57. Caplen, H., Peters, C. J. \& Bishop, D. H. Mutagen-directed attenuation of Rift Valley fever virus as a method for vaccine development. J. Gen. Virol. 66, 2271-2277 (1985). (Pt 10).

58. Phoenix, l., Lokugamage, N., Nishiyama, S. \& Ikegami, T. Mutational analysis of the Rift Valley fever virus glycoprotein precursor proteins for $\mathrm{Gn}$ protein expression. Viruses 8, pii: E151 (2016).

59. Hemati, B. et al. Bluetongue virus targets conventional dendritic cells in skin lymph. J. Virol. 83, 8789-8799 (2009).

60. Drosten, C. et al. Rapid detection and quantification of RNA of Ebola and Marburg viruses, Lassa virus, Crimean-Congo hemorrhagic fever virus, Rift Valley fever virus, dengue virus, and yellow fever virus by real-time reverse transcription-PCR J. Clin. Microbiol. 40, 2323-2330 (2002).

61. Pages, N. et al. Culicoides midge bites modulate the host response and impact on bluetongue virus infection in sheep. PLoS One 9, e83683 (2014).

C Open Access This article is licensed under a Creative Commons Attribution 4.0 International License, which permits use, sharing adaptation, distribution and reproduction in any medium or format, as long as you give appropriate credit to the original author(s) and the source, provide a link to the Creative Commons license, and indicate if changes were made. The images or other third party material in this article are included in the article's Creative Commons license, unless indicated otherwise in a credit line to the material. If material is not included in the article's Creative Commons license and your intended use is not permitted by statutory regulation or exceeds the permitted use, you will need to obtain permission directly from the copyright holder. To view a copy of this license, visit http://creativecommons. org/licenses/by/4.0/.

(c) The Author(s) 2018 